\title{
Axiomatics for the external numbers of nonstandard analysis
}

\author{
BRUNO DINIS \\ IMME VAN DEN BERG
}

\begin{abstract}
Neutrices are additive subgroups of a nonstandard model of the real numbers. An external number is the algebraic sum of a nonstandard real number and a neutrix. Due to the stability by some shifts, external numbers may be seen as mathematical models for orders of magnitude. The algebraic properties of external numbers gave rise to the so-called solids, which are extensions of ordered fields, having a restricted distributivity law. However, necessary and sufficient conditions can be given for distributivity to hold. In this article we develop an axiomatics for the external numbers. The axioms are similar to, but mostly somewhat weaker than the axioms for the real numbers and deal with algebraic rules, Dedekind completeness and the Archimedean property. A structure satisfying these axioms is called a complete arithmetical solid. We show that the external numbers form a complete arithmetical solid, implying the consistency of the axioms presented. We also show that the set of precise elements (elements with minimal magnitude) has a built-in nonstandard model of the rationals. Indeed the set of precise elements is situated between the nonstandard rationals and the nonstandard reals whereas the set of non-precise numbers is completely determined.
\end{abstract}

2010 Mathematics Subject Classification 03H05 (primary); 03C65, 26E35 (secondary)

Keywords: External numbers, axiomatics, nonstandard analysis, complete arithmetical solids

\section{Introduction}

Consider a nonstandard model of the real number system ${ }^{*} \mathbb{R}$. A neutrix is an additive convex subgroup of ${ }^{*} \mathbb{R}$ and an external number is the algebraic sum of a nonstandard real number with a neutrix. In such a nonstandard framework there are many neutrices such as $\oslash$, the external set of all infinitesimals, and $£$, the external set of all limited numbers, ie numbers bounded in absolute value by a standard number.

Typically external numbers are subsets of ${ }^{*} \mathbb{R}$ having neither infimum nor supremum, being stable for some translations, additions and multiplications. As argued in Koudjeti 
and Van den Berg [20] and Dinis and Van den Berg [11], they are models of orders of magnitude or transitions with imprecise boundaries of Sorites type (Dinis [9]). With external numbers it is possible to work directly with imprecisions and errors without recourse to upper bounds. They generate a calculus of propagation of errors not unlike the calculus of real numbers, allowing for total order and even for a sort of generalized Dedekind completeness property. Some applications in asymptotics, singular perturbations, linear algebra and statistics are contained in [20], Justino and Van den Berg [15] and Van den Berg [3], and the references mentioned in the latter article. The term neutrix is borrowed from Van der Corput [7]. His neutrices are rings of "neglectable functions". The calculation rules satisfied by the external numbers are significantly stronger than the functional asymptotic calculus of $o$ 's and $O$ 's de Bruijn [5] and Van der Corput's neutrices which for instance do not respect total order.

Algebraic properties of external numbers have been studied in Koudjeti [19], [20] and [11]. Respecting total order, they are based on semigroup operations more than group operations, a sort of "mellowed" version of the common rules of calculation of real numbers. In Dinis and Van den Berg [12] it was shown that the set of cosets of non-Archimedean ordered fields with respect to all possible convex subgroups for addition has a similar algebraic structure. Such structures were called solids. Elements of a solid are the sum of a precise element and a magnitude. The magnitudes act as individualized neutral elements and correspond to the convex subgroups, and the precise elements, ie elements with magnitude "zero", to the elements of the underlying ordered field. Solids are not completely distributive, but necessary and sufficient conditions can be given for triples of elements to satisfy distributivity (Dinis and Van den Berg [10]).

In this article we extend the axiomatic laws of solids. The axioms added include multiplicative properties of neutrices, a generalized Dedekind completeness property, and an Archimedean property. The multiplicative axioms are inspired by the results of [19] and [20] on neutrices which are idempotent for multiplication. The axioms determine the product of idempotent neutrices, in fact of all neutrices, because it is postulated that every neutrix is a multiple of an idempotent neutrix. In $Z F C$ the structure of real numbers $\mathbb{R}$ is characterized up to isomorphism in a second-order language, as the unique Dedekind complete ordered field, or equivalently as the unique Archimedean complete ordered field in which Cauchy sequences converge. However second-order properties of nonstandard models of the reals are less obvious. Therefore we intend to remain in a first-order language and so the generalized Dedekind completeness axiom is stated in the form of a scheme. In order to deal with the Archimedean property we assume some Peano-like axioms, including a scheme on induction. Due to this Archimedean property models must include a copy of the nonstandard integers, hence 
of the nonstandard rationals, and due to the generalized Dedekind completeness it must be possible to embed models in the nonstandard reals.

We prove consistency of the axiomatics in the setting of a nonstandard model ${ }^{*} \mathbb{R}$, which has the form of an adequate ultralimit for a bounded version of Nelson's syntactical Reduction Algorithm [23] to hold. Admissible models will be called complete arithmetical solids. Up to isomorphism, once the magnitudes are specified the set of non-precise numbers of a complete arithmetical solid is completely determined as sums of a nonstandard rational and a magnitude. For the set of precise elements of a model we give upper and lower bounds, in fact the precise elements are situated between the nonstandard rationals and the nonstandard reals. As a result, complete arithmetical solids come closer to a syntactical characterization of the external numbers than the solids of [10] which can also be built on non-Archimedean ordered fields.

There have been various attempts to deal with the external algebraic and order structure of the real line. Wattenberg [29] and Gonshor [14] developed a calculus of the lower halflines of the nonstandard real line. However this gave rise to a less rich algebraic structure, and also they do not consider completeness properties of Dedekind kind. Keisler and Schmerl [18] consider two other completeness properties of the external real line in a model-theoretic setting, Scott completeness and Bolzano-Weierstrass completeness, without developing an algebraic calculus. From an axiomatic point of view Scott completeness and Bolzano-Weierstrass completeness were reconsidered in Kanovei and Reeken [16].

For sake of clarity and reference we start in Section 2 with an overview of all the axioms.

The axiom scheme on Generalized Dedekind completeness is discussed in Section 3. It is stated in terms of precise numbers, but typically concerns halflines which are not precise, ie stable under some shifts. For precise definable halflines Generalized Dedekind completeness reduces to ordinary Dedekind completeness, ie halflines including the extremum, and halflines with the extremum just beyond. When applied to halflines of a solid (thus including non-precise numbers), three types of halflines occur, instead of two; it is shown that they are mutually exclusive.

With Generalized Dedekind completeness one can define the minimal magnitude including unity, denoted by $£$, and the maximal magnitude without unity, denoted by $\oslash$. Section 4 shows that their algebraic properties correspond to a large extent to the properties of the limited numbers, respectively the infinitesimals in a nonstandard model of the reals. However they are somewhat weaker, and it is not possible to decide whether $\oslash £=\oslash$ or $\oslash £=£$. In Section 5 we choose the product of $\oslash$ and $£$ to be $\oslash$, in accordance with the fact that the product of an infinitesimal and limited real is 
infinitesimal. The axiom is stated in terms of the product of an idempotent magnitude with unity and its maximal ideal. Ideals are defined by analogy to ideals of rings, and the existence of maximal ideals follows from Generalized Dedekind completeness. An axiom that says that an arbitrary magnitude is a multiple of an idempotent magnitude enables to settle the product of any two neutrices.

In Section 6 we show relative consistency of our axiomatics with $Z F C$ by the construction of a model based on external subsets of an appropriate nonstandard model of the reals.

In Section 7 we show that, up to isomorphism, the precise elements of a model are situated between the nonstandard rationals and the nonstandard reals. In a sense, it is also possible to identify a notion of standard part, here called shadow. It is shown that the set of shadows of the precise elements is situated between the set of shadows of the nonstandard rationals and the set of shadows of the nonstandard reals. Finally we investigate the relation between the standard structure and the nonstandard structure of a complete arithmetical solid. More precisely, we show that the Leibniz rules hold for the precise elements of a complete arithmetical solid, ie the precise elements are a model of the axiomatics ZFL (Lutz [22] and Callot [6]), and that the "natural numbers" in a solid are a model for the axiomatics given by Nelson in [24] here called REPT, but with external induction restricted to the language $\{+, \cdot\}$. These "weak" nonstandard axiomatics are not without interest. Indeed, in [6] Callot showed that a substantial part of ordinary analysis can be carried out in ZFL and in [24] Nelson argued that REPT is sufficient for advanced stochastics.

In this way, our axiomatic approach gives rise to an alternative way to build nonstandard real numbers in which, unlike Nelson's approaches [23], [24] the infinitesimals are not postulated through a new undefined symbol, but by the existence of magnitudes. It does not have the force of REPT but has the advantage of being able to deal with some external sets. Indeed, complete arithmetical solids in a sense incorporate certain external sets in the form of elements, in particular external sets relevant for calculatory aspects. Our approach enters also in the tradition of the usual axiomatic presentation of the real numbers by field axioms, an axiom on completeness, and possibly an axiom on the Archimedean property.

\section{The Axioms}

The axioms will come in groups. The first group deals with algebraic properties. The algebraic axioms consist of axioms for addition, axioms for multiplication, axioms for 
the order, axioms relating addition and multiplication, axioms of existence guaranteeing among other things that models are richer than fields and axioms on the value of products of magnitudes. Then we present an axiom scheme on a generalized Dedekind completion and finally a group of axioms, including a scheme, on natural numbers and the Archimedean property.

We will present the axioms in a first-order language. Addition, multiplication and order will be presented in the language $\{+, \cdot, \leq\}$, later on we add a unary predicate $N$ to deal with natural numbers. Neutrices are represented by magnitudes which are individualized neutral elements. As such the individualized neutral elements are unique. The proof is similar to the proof of the uniqueness of neutral elements in groups (see Dinis and Van den Berg [11]). Often it is convenient to use the functional notation $e(x)$ to indicate the individualized neutral element of the element $x$. The individualized neutral elements for multiplication (unities) are also unique and we may use the functional notation $u(x)$. With respect with the individualized neutral element the symmetrical element is also unique. We may denote it by $s(x)$ or $-x$ in the case of addition and $d(x)$ or $1 / x$ in the case of multiplication.

\subsection{Algebraic axioms}

Axioms for addition and multiplication are similar and gave rise to the notion of assembly in [11]. An assembly is a completely regular semigroup (union of groups), in which the magnitude operation is linear. A structure satisfying Axioms $2.1-2.29$ was called a solid in Dinis and Van den Berg [12].

\section{Axioms for addition.}

Axiom 2.1 $\forall x \forall y \forall z(x+(y+z)=(x+y)+z)$

Axiom 2.2 $\forall x \forall y(x+y=y+x)$

Axiom 2.3 $\forall x \exists e(x+e=x \wedge \forall f(x+f=x \rightarrow e+f=e))$

Axiom 2.4 $\forall x \exists s(x+s=e(x) \wedge e(s)=e(x))$

Axiom 2.5 $\forall x \forall y(e(x+y)=e(x) \vee e(x+y)=e(y))$

It follows from Axioms 2.3 and 2.5 that $e(x+y)=e(x)+e(y)$, ie the magnitude operation is linear. 


\section{Axioms for multiplication}

Axiom 2.6 $\forall x \forall y \forall z(x(y z)=(x y) z)$

Axiom 2.7 $\forall x \forall y(x y=y x)$

Axiom 2.8 $\forall x \neq e(x) \exists u(x u=x \wedge \forall v(x v=x \rightarrow u v=u))$

Axiom 2.9 $\forall x \neq e(x) \exists d(x d=u(x) \wedge u(d)=u(x))$

Axiom 2.10 $\forall x \neq e(x) \forall y \neq e(y)(u(x y)=u(x) \vee u(x y)=u(y))$

Again we have $u(x y)=u(x) u(y)$.

\section{Order axioms}

Axioms 2.11-2.14 state that " $\leq$ " is a total order relation. Axiom 2.15 states that the order relation is compatible with addition. The last two axioms state that the order relation is compatible with the multiplication by positive elements. With respect with classical order axioms, essentially the only new axiom is Axiom 2.16. This axiom states that if an element is "small", in the sense that it gets absorbed when added to a certain magnitude, then it is also smaller than that magnitude in terms of the order.

Axiom 2.11 $\forall x(x \leq x)$

Axiom 2.12 $\forall x \forall y(x \leq y \wedge y \leq x \rightarrow x=y)$

Axiom 2.13 $\forall x \forall y \forall z(x \leq y \wedge y \leq z \rightarrow x \leq z)$

Axiom 2.14 $\forall x \forall y(x \leq y \vee y \leq x)$

Axiom 2.15 $\forall x \forall y \forall z(x \leq y \rightarrow x+z \leq y+z)$

Axiom 2.16 $\forall x \forall y(y+e(x)=e(x) \rightarrow(y \leq e(x) \wedge-y \leq e(x)))$

Axiom 2.17 $\forall x \forall y \forall z((e(x)<x \wedge y \leq z) \rightarrow x y \leq x z)$

Axiom 2.18 $\forall x \forall y \forall z((e(y) \leq y \leq z) \rightarrow e(x) y \leq e(x) z)$

It follows from Axiom 2.16 that for magnitudes the order can be characterized in terms of addition. Indeed, for all $x, y$ one has $e(x) \leq e(y)$ if and only if $e(x)+e(y)=e(y)$. 


\section{Axioms concerning addition and multiplication}

The first three axioms state properties of magnitudes. Axiom 2.19 states that the product of an element and a magnitude is a magnitude. Axiom 2.20 gives the magnitude of the product and Axiom 2.21 the magnitude of the individualized unity. Axiom 2.22 states that the distributive law holds up to a magnitude. Due to this restriction one needs to specify the symmetrical element of the product of two elements as done in Axiom 2.23.

Axiom 2.19 $\forall x \forall y \exists z(e(x) y=e(z))$

Axiom 2.20 $\forall x \forall y(e(x y)=e(x) y+e(y) x)$

Axiom 2.21 $\forall x \neq e(x)(e(u(x))=e(x) d(x))$

Axiom 2.22 $\forall x \forall y \forall z(x y+x z=x(y+z)+e(x) y+e(x) z)$

Axiom 2.23 $\forall x \forall y(-(x y)=(-x) y)$

\section{Axioms of existence}

Axioms 2.24 gives the existence of a minimal magnitude which we will denote by 0 . Elements $p$ such that $e(p)=0$ are called precise. Axiom 2.25 gives the existence of a minimal unity which we denote by 1 . Axiom 2.26 states that there exists a maximal magnitude $M$, in fact, when constructing a model it corresponds to its domain. Axiom 2.27 states that there exist magnitudes other than 0 and $M$, implying that the domain of the model can no longer be a field. Axiom 2.28 states that any element is the sum of a precise element and a magnitude. Axiom 2.29 states that two magnitudes are separated by an element which is not a magnitude. Such an element is called zeroless. It follows from the existence of zeroless elements that $1 \neq 0$, hence also that a solid must contain a copy of $\mathbb{Q}$.

Axiom 2.24 $\exists m \forall x(m+x=x)$

Axiom 2.25 $\exists u \forall x(u x=x)$

Axiom 2.26 $\exists M \forall x(e(x)+M=M)$

Axiom 2.27 $\exists x(e(x) \neq 0 \wedge e(x) \neq M)$

Axiom 2.28 $\forall x \exists a(x=a+e(x) \wedge e(a)=0)$ 
Axiom 2.29 $\forall x \forall y(x=e(x) \wedge y=e(y) \wedge x<y \rightarrow \exists z(z \neq e(z) \wedge x<z<y))$

\section{Axioms on the product of magnitudes}

Next axiom needs some preparatory definitions. A magnitude $e$ is idempotent if $e e=e$. Let $y$ be an idempotent magnitude such that $1<y$. An ideal $z$ of $y$ is a magnitude such that $z \leq y$ and $\forall p(e(p)=0 \wedge 0 \leq p<y \rightarrow p z \leq z$. An ideal $x$ of $y$ is maximal if $x<y$ and all ideals $z$ such that $x \leq z \leq y$ satisfy $z=x$ or $z=y$.

In a semantic setting, the ideals defined above are elements and not sets, such as in the usual algebraic interpretation of ideals of a ring. As will be shown, the two notions of ideal are closely related. Maximal ideals happen to be idempotent. The existence of maximal ideals in the setting of rings is equivalent to the Axiom of Choice. The existence of maximal ideals in terms of magnitudes will be a consequence of Axiom 2.32 below.

By Axiom 2.19 the product of magnitudes is a magnitude. The value of the product is obtained by relating them to idempotent magnitudes. Axiom 2.31 states that a magnitude is the product of a precise element and an idempotent magnitude. As it turns out, the value of all products of idempotent magnitudes is determined by Axiom 2.30.

Axiom 2.30 Let $y$ be an idempotent magnitude such that $1<y$ and $x$ be the maximal ideal of $y$. Then $x y=x$.

Axiom 2.31 $\forall x(x=e(x) \rightarrow \exists p \exists y(e(p)=0 \wedge y=e(y) \wedge y y=y \wedge x=p y))$

\subsection{Generalized Completeness axiom}

The axiom on generalized Dedekind completeness comes in the form of a scheme, for a definite class of formulas.

Let $k$ be a natural number. Let $\Phi\left(x_{1}, \ldots, x_{k}\right)$ be a formula of the language $\{+, \cdot, \leq\}$ with free variables $x_{1}, \ldots, x_{k}$. The formula $\Phi$ is called restricted if each quantifier ranges over precise elements.

Axiom 2.32 states that a lower halfline defined by a restricted formula $A(x)$ of a free precise variable $x$ has a lowest upper bound which is the sum of a precise element and a magnitude $e$. This magnitude corresponds to the set of precise elements which leave the halfline invariant under addition. We will extend the completeness property in Section 3 to halflines of non-precise elements. As will be shown this generates three types of halflines instead of two. 
Axiom 2.32 (Generalized Dedekind completeness) Let $A$ be a restricted formula (possibly with non-precise parameters) allowing for a free precise variable $x$, and such that

$$
\exists x A(x) \wedge \forall x \forall y(A(x) \wedge y<x \rightarrow A(y)) .
$$

Then one of the following holds:

(1) $\exists \sigma \forall x(A(x) \leftrightarrow x \leq \sigma)$

(2) $\exists \tau \forall x(A(x) \leftrightarrow \forall t(t+e(\tau)=\tau \rightarrow x<t)$

It will be shown that 1 and 2 are mutually exclusive, and that $\sigma$ and $\tau$ are unique. They are called the weak least upper bound of $A$ and are denoted by zup $A$. Condition (1) expresses the lower-halfline property. If $A$ is an arbitrary non-empty property, one may define $A^{\prime}$ by

$$
A^{\prime}(x) \leftrightarrow \exists y(e(y)=0 \wedge x \leq y \wedge A(y))
$$

Then $A^{\prime}$ satisfies (1). We extend the notion of weak supremum by $\operatorname{defining} \operatorname{zup} A=$ zup $A^{\prime}$. Working with upper halflines one may define in a similar way a weak greatest lower bound winf. It will be seen that both notions can be appropriately extended to restricted formulas of a non-precise variable. We use this possibility in the following.

We define $\Phi(e)$, respectively $\Psi(f)$, by:

$$
\begin{aligned}
& e+e=e \wedge e<1 \\
& f+f=f \wedge 1<f
\end{aligned}
$$

Then we define:

$$
\begin{gathered}
\oslash=\operatorname{zup} \Phi \\
£=\operatorname{winf} \Psi
\end{gathered}
$$

So $£$ is the minimal magnitude greater than 1 and $\oslash$ is the maximal magnitude less than 1 . We will see in Section 4 that $£$ and $\oslash$ are idempotent and that $\oslash$ is the maximal ideal of $£$ in the sense of Axiom 2.30. It results from this axiom that $\oslash £=\oslash$.

\subsection{Arithmetical axioms}

The last group of axioms allows to distinguish between non-Archimedean ordered structures and structures with a (nonstandard) archimedean property. We extend the language with a symbol $N$ which is an unary predicate allowing for a free precise variable $x$. The symbol $N$ is intended to represent the natural numbers. In this sense 
Axiom 2.33 states that there are no negative natural numbers, 0 is a natural number, the successor of a natural number is a natural number, and that these are consecutive indeed, ie between a natural number and its successor there is no other natural number.

Axiom 2.33 (Natural numbers)

$$
\begin{aligned}
& \forall x(x<0 \rightarrow \neg N(x)) \wedge N(0) \\
& \quad \wedge \forall x(N(x) \rightarrow \forall y(x<y<x+1 \rightarrow \neg N(y)) \wedge N(x+1))
\end{aligned}
$$

It is clear that induction does not hold for all formulas. Indeed, $0<£$ and if $x<£$, then $x+1<£$, but there are elements $x$ such that $£<x$. It is well-known that within nonstandard analysis one can only apply induction to the so-called internal formulas in the sense of [23]. This means in our context that all parameters must be natural numbers and also that all references to non-precise elements such as $£$ and $\oslash$ must be banned. To do so we allow induction in one precise variable, only for properties with quantifications over precise variables and with natural numbers as possible parameters.

Axiom 2.34 (Induction) Let $A$ be a property expressed with the symbols $0,1,+$, and $\leq$, allowing for a free precise variable $x$ and quantifications only over precise variables with all its parameters $y$ satisfying $N(y)$. Then

$$
(A(0) \wedge \forall x(N(x) \rightarrow(A(x) \rightarrow A(x+1))) \rightarrow \forall x(N(x) \rightarrow A(x))) .
$$

In Section 7 it is shown that for a larger class of formulas induction holds over the natural numbers less than $£$.

The last axiom states the Archimedean property for the natural numbers given by Axiom 2.33 .

Axiom 2.35 (Archimedean property)

$$
\forall x \forall y(0<x<y<M \rightarrow \exists z(N(z) \wedge z x>y))
$$

A set $S$ satisfying all the axioms given above will be called a complete arithmetical solid.

\section{Generalized Dedekind completeness}

Dedekind completeness is the property that every Dedekind cut of the real numbers is generated by a real number, corresponding to the intuition that the real line has no 
"gaps". Axiom 2.32 gives a generalization of the Dedekind completeness property. Because it is not excluded that some nonstandard models of the real line do have gaps (Keisler and Schmerl [18]), it is written in the form of an axiom scheme in a first-order language, ie without referring to subsets. The axiom scheme is stated for properties of precise elements, defined by a restricted formula; in fact it can be extended to such properties of non-precise elements. As we will see this generates three types of halflines instead of two.

We will call a solid complete if it satisfies the Axioms 2.1-2.32. Let $S$ be a complete solid. Let $A$ be a formula of the variable $x$. By a matter of convenience we identify $A$ with its interpretation in the set $S$. So $x \in A$ is the interpretation of $A(x)$ and $x \notin A$ the interpretation of $\neg A(x)$.

Definition 3.1 Let $S$ be a complete solid and let $\emptyset \neq A, B \subseteq S$. Then $A$ is said to be a lower halfline if $x \in A$ and $y<x$ imply that $y \in A$ and $B$ is said to be an upper halfline if $x \in B$ and $x<y$ imply that $y \in B$. A lower halfline $A$ is precise if there is no precise positive $d$ such that $a+d \in A$ for all $a \in A$.

Remark If $A \neq S$ is a lower halfline, then $B=S \backslash A \neq \emptyset$ is an upper halfline and vice-versa.

Theorem 3.2 If $A$ is a lower halfline defined by a restricted formula in a complete solid $S$, it has one of the following forms:

(1) $\exists \rho \forall x(x \in A \leftrightarrow x \leq \rho)$

(2) $\exists \sigma \forall x(x \in A \leftrightarrow x<\sigma)$

(3) $\exists \tau \forall x(x \in A \leftrightarrow \forall t(t+e(\tau)=\tau \rightarrow x<t)$

In order to prove Theorem 3.2 we will make Axiom 2.29 more operational, by showing that two magnitudes can always be separated by a precise element. Also the elements $t$ of Axiom 2.32.2 and Theorem 3.2.3 may be taken precise. We prove also some other properties on separation by a precise element.

Proposition 3.3 In Axiom 2.32.2 the elements $t$ may be taken precise.

Proof Let $t$ be such that $t+e(\tau)=\tau$. Then $t=p+e(t)$ with $e(p)=0$. Because $e(t) \leq e(\tau)$, one has $p+e(\tau)=p+e(t)+e(\tau)=t+e(\tau)=\tau$.

Let $x$ be precise. Suppose that $x<t$ for all $t$ such that $t+e(\tau)=\tau$. Then it holds in particular for $t=p$ with $p$ precise. Conversely, suppose that $x<p$, for all precise $p$ 
such that $\tau=p+e(\tau)$. Let $t$ be such that $t+e(\tau)=\tau$. Then there exists a precise $q$ such that $t=q+e(t)$. Then $q+e(\tau)=\tau$. Hence $x<q \leq q+e(t)=t$. This implies that the two criteria are equivalent.

Proposition 3.4 In Theorem 3.2.3 the elements $t$ may be taken precise.

The proof is analogous to the proof of Proposition 3.3.

Lemma 3.5 (Dinis and Van den Berg [10, Proposition 2.10]) Let $A$ be an ordered assembly and let $x, y \in A$. If $x<e(x)$, then $x<e(y)$.

Proof Assume $x<e(x)$. We suppose towards a contradiction that $e(y) \leq x$. Then $e(y)<e(x)$, so $e(x) \leq e(y)+e(x) \leq e(x)+e(x)=e(x)$. Hence $e(x)=e(y)+e(x) \leq$ $x+e(x)=x$, a contradiction.

Lemma 3.6 Let $S$ be a solid and let $x, z \in S$. If $e(x)<z$ and $z$ is zeroless, there is a precise element $t$ such that $e(x)<t \leq t+e(z)<z$.

Proof Let $z=p+e(z)$ with $p$ precise. If $z<e(z)$ then $z<e(x)$ by Lemma 3.5, a contradiction. Hence $e(z)<z$, meaning that $p$ is positive, so $0<p / 2<p$. One has $e(z)<p / 2$. Indeed, if $p / 2 \leq e(z)$ one would have $p \leq 2 e(z)=e(z)$ which is a contradiction. It follows that $p / 2+e(z)<p \leq p+e(z)=z$. If $e(x) \leq e(z)$, we are done. If $e(z)<e(x)$, suppose that $p / 2 \leq e(x)$. Then $p \leq 2 e(x)=e(x)$ and $z=p+e(z) \leq e(x)+e(x)=e(x)$, a contradiction. Hence $e(x)<p / 2 \leq p / 2+e(z)$.

Lemma 3.7 The element $z$ in Axiom 2.29 may be supposed precise.

Proof Let $z$ be zeroless and such that $e(x)<z<e(y)$. By Lemma 3.6 there exists a precise $t$ such $e(x)<t<z<e(y)$.

Lemma 3.8 Let $S$ be a solid and $x, y \in S$ be such that $x<y$. Then there exists a precise element $p$ such that $x<p<y$.

Proof Without restriction of generality we may assume that $x=e(x)$. The case $y=e(y)$ follows from Lemma 3.7 and the case where $y$ is zeroless follows from Lemma 3.6.

Lemma 3.9 Let $S$ be a solid and $x, y \in S$ be such that $\forall t(e(t)=0 \wedge t+e(y)=$ $y \rightarrow x<t)$. Then there exists a precise element $p$ such that $x<p$ and $\forall t(e(t)=$ $0 \wedge t+e(y)=y \rightarrow p<t)$. 
Proof Without restriction of generality we may assume that $x=e(x)$. Then $y$ is zeroless. Let $q$ be precise such that $y=q+e(y)$. Put $p=q / 2$. Then $e(x)<p$, otherwise $q \leq 2 e(x)=e(x)$, a contradiction. Also $e(y)<p$, otherwise $q \leq 2 e(y)=e(y)$ and $y$ would not be zeroless. Let $t$ be precise such that $t+e(y)=y$. Then $e(y)<t$, otherwise $y$ would not be zeroless. Suppose $t \leq p$. Then $y=t+e(y)<t+t \leq q$, a contradiction. Hence $p<t$.

Proof of Theorem 3.2 Let $A$ be a lower halfline defined by a restricted formula in a complete solid $S$. We define $\hat{A}$ by $\hat{A}=\{x \in A \mid e(x)=0\}$. Then also $\hat{A}$ is defined by a restricted formula. By Axiom 2.32 and Proposition 3.3, either there exists $\alpha \in S$ such that, whenever $p$ is precise, one has $p \in A$ if and only if $p \leq \alpha$, or there exists $\beta$ such that whenever $p$ is precise, one has $p \in A$ if and only if $p<q$ for all precise $q$ such that $q+e(\beta)=\beta$.

As for the first case, we distinguish the subcases $\alpha \in A$ and $\alpha \notin A$. Assume $\alpha \in A$. Let $x \in A$. Suppose $\alpha<x$. By Lemma 3.8 there exists a precise element $r \in A$ such that $\alpha<r<x$, a contradiction. Hence $x \leq \alpha$. Conversely, assume $x \leq \alpha$. Then $x \in A$ by the definition of lower halfline. Taking $\rho=\alpha$ we obtain $x \in A$ if and only if $x \leq \rho$.

Assume now that $\alpha \notin A$. One proves as above that if $x \in A$ then $x \leq \alpha$, in fact, $x<\alpha$ because $\alpha \notin A$. Conversely, assume $x<\alpha$. By Lemma 3.8 there exists a precise element $r$ such that $x<r<\alpha$. Then $r \in \hat{A} \subseteq A$. Hence $x \in A$. Taking $\sigma=\alpha$ we obtain $x \in A$ if and only if $x<\sigma$.

As for the second case, assume first that $x \in A$. Suppose that there exists a precise $t$ such that $t<x$ and $t+e(\beta)=\beta$. We may write $x=p+e(x)$ with $p$ precise and $t<p$. Now $p<t$ because $p \in \hat{A}$, a contradiction. Hence $x<t$. Finally assume that $x<t$ for all precise $t$ such that $t+e(\beta)=\beta$. By Lemma 3.9 there exists a precise $p$ such that $x<p$ and $p<t$ for all precise $t$ such that $t+e(\beta)=\beta$. Then $p \in \hat{A} \subseteq A$, hence $x \in A$ because $A$ is a lower halfline. Taking $\tau=\beta$, we conclude that $x \in A$ if and only if $\forall t(e(t)=0 \wedge t+e(\tau)=\tau \rightarrow x<t)$. By Proposition 3.4 this is equivalent to $\forall t(t+e(\tau)=\tau \rightarrow x<t)$.

If $A$ is a precise lower halfline the case 3.2.3 reduces to the case 3.2.2, so the Generalized Dedekind completeness of Axiom 2.32 and Theorem 3.2 correspond to ordinary Dedekind completeness.

Proposition 3.10 If $A$ is precise, in the third case of the criterion in Theorem 3.2 the element $\tau$ is precise. In fact the criterion is equivalent to

$$
\exists \tau(e(\tau)=0 \wedge \forall x(x \in A \leftrightarrow x<\tau) .
$$


Proof Suppose that $0<e(\tau)$. Let $p$ be precise and such that $0<p<e(\tau)$. Let $x \in A$. If there exists $t$ such that $t+e(\tau)=\tau$ and $t \leq x+p$, then $\tau \leq x+p+e(\tau)=x+e(\tau)$. Note that $x+e(\tau) \leq t+e(\tau)=\tau$. Hence $x+e(\tau)=\tau$, which means that $x<x$, a contradiction. Hence $x+p \in A$, but this means that $A$ is not precise, again a contradiction. Hence $e(\tau)=0$ and $\tau$ is precise. In addition, if $t$ is such that $t+e(\tau)=\tau$, then $t=\tau$

If the lower halfline $A$ is not precise, the three cases may indeed occur and are mutually exclusive. We will call the elements $\rho, \sigma$ and $\tau$ weak least upper bounds, denoted by zup $A$. Moreover, the elements $\rho, \sigma$ and $\tau$ are unique and we write $A=(-\infty, \rho]$, $A=(-\infty, \sigma)$ and $A=(-\infty, \tau[[$ respectively. In the first case the halfline is called closed, and $\rho$ may be called the maximum of $A$, written $\rho \equiv \max A$. In the second case the halfline is called open and $\sigma$ is an ordinary least upper bound, which we may call the supremum of $A$, written $\sigma \equiv \sup A$. In the third case we call the halfline strongly open (see also Van den Berg [4]) and $\tau$ the weak supremum of $A$. We may define weak least upper bounds for any set $A$ by defining zup $A \equiv \operatorname{zup} \bar{A}$ where $\bar{A} \equiv\{x \in S \mid \exists a \in A(x \leq a)\}$.

Theorem 3.11 With respect to Theorem 3.2 the elements $\rho, \sigma$ and $\tau$ are unique, and the cases 1 and 2, and the cases 1 and 3 are mutually exclusive. If $A$ is a precise lower halfline the third case reduces to the second case. If $A$ is not precise the three cases are mutually exclusive. Moreover, $\rho \in A$ and $\sigma, \tau \notin A$.

Proof Clearly $\rho \in A$ and $\sigma \notin A$, because $\rho \leq \rho$ and $\sigma \nless \sigma$. Suppose towards a contradiction that $\tau \in A$. Then for all $t$ such that $t+e(\tau)=\tau$ one has $\tau<t$. Then $\tau<t+e(\tau)=\tau$, a contradiction. Hence $\tau \notin A$.

To show that $\rho$ is unique suppose that $\rho^{\prime}$ is such that $x \in A$ if and only if $x \leq \rho$ and if and only if $x \leq \rho^{\prime}$. Then $\rho, \rho^{\prime} \in A$, hence $\rho \leq \rho^{\prime}$ and $\rho^{\prime} \leq \rho$. One concludes that $\rho$ is unique by Axiom 2.12.

To show that $\sigma$ is unique suppose that $\sigma^{\prime}$ is such that $x \in A$ if and only if $x<\sigma$ if and only if $x<\sigma^{\prime}$. If $\sigma<\sigma^{\prime}$ then $\sigma \in A$ hence $\sigma<\sigma$ which is absurd. If $\sigma^{\prime}<\sigma$ then similarly $\sigma^{\prime} \in A$ and $\sigma^{\prime}<\sigma^{\prime}$, which is absurd. Hence $\sigma=\sigma^{\prime}$ by Axiom 2.14.

In order to show that $\tau$ is unique, suppose that $x \in A$ if and only if $x<t$ for all precise $t$ with $t+e(\tau)=\tau$, and also if and only if $x<t^{\prime}$ for all precise $t^{\prime}$ with $t^{\prime}+e\left(\tau^{\prime}\right)=\tau^{\prime}$. Assume that $\tau<\tau^{\prime}$. We may suppose that $\tau=e(\tau)$. Suppose first that $\tau^{\prime}=e\left(\tau^{\prime}\right)$. Then by Lemma 3.6 there is a precise element $p$ such that $e(\tau)<p<\tau^{\prime}$. Then $-p<t$ for all precise $t$ such that $t+e(\tau)=\tau$, otherwise $p+t \leq 0 \leq e(\tau)$, 
so $p \leq-t+e(\tau)=-(t-e(\tau))=-(t+e(\tau))=-e(\tau)=e(\tau)$, which is a contradiction. Hence $-p \in A$. On the other hand $-p+e\left(\tau^{\prime}\right)=-\left(p-e\left(\tau^{\prime}\right)\right)=$ $-\left(p+e\left(\tau^{\prime}\right)\right)=-e\left(\tau^{\prime}\right)=e\left(\tau^{\prime}\right)$. Hence $-p \notin A$, a contradiction. Secondly, we suppose that $\tau^{\prime}$ is zeroless. It follows from Lemma 3.6 that there exists a precise $p$ such that $e(\tau)<p+e\left(\tau^{\prime}\right)<\tau^{\prime}$. By the second inequality $p \in A$ and by the first inequality $p \notin A$, a contradiction. Hence $\tau^{\prime} \leq \tau$. Similarly one shows that $\tau \leq \tau^{\prime}$. Hence $\tau=\tau^{\prime}$.

We prove next that the cases 3.2.1 and 3.2.2 are mutually exclusive. If not, because $\rho \in A$ one has $\rho<\sigma$. By Lemma 3.6 there is a precise element $s$ such that $\rho<s<\sigma$. Then $s \notin A$ because $\rho<s$, and $s \in A$ because $s<\sigma$, a contradiction. Hence the cases 3.2.1 and 3.2.2 are mutually exclusive.

We prove next that the cases 3.2.1 and 3.2.3 are mutually exclusive. If not, because $\rho \in A$, for all $t$ such that $t+e(\tau)=\tau$ one has $\rho<t$. By Lemma 3.9 there is a precise $p$ such that $\rho<p$ and $p<t$ for all $t$ such that $t+e(\tau)=\tau$. Then both $p \notin A$ and $p \in A$, a contradiction.

Finally we relate the cases 3.2.2 and 3.2.3. By Proposition 3.10 they coincide if $A$ is precise. Assume that $A$ is not precise. If $\sigma<\tau$, we obtain a contradiction along the lines of the previous case. Suppose $\tau<\sigma$. By Lemma 3.8 there exists a precise $q$ such that $\tau<p<\sigma$. Then both $p \notin A$ and $p \in A$, a contradiction. Hence $\sigma=\tau$. Hence the cases 3.2.2 and 3.2.3 are mutually exclusive.

As for upper halflines, the definition of weak greatest lower bounds winf is similar, but not entirely analogous, to the definition of weak least upper bounds, according to the three possibilities mentioned in the following theorem.

Theorem 3.12 Let $S$ be a complete solid and $B \subseteq S$ be an upper halfline defined by a restricted formula. Then $B$ has one of the following forms:

(1) $\exists \rho \forall y(y \in B \leftrightarrow \rho \leq y)$

(2) $\exists \sigma \forall y(y \in B \leftrightarrow \sigma<y)$

(3) $\exists \tau \forall y(y \in B \leftrightarrow \exists t(t+e(\tau)=\tau \wedge t \leq y))$

Proof The case $B=S$ corresponds to 3.12.3. If $B \subset S$, define $A=S \backslash B$. Then $A$ is a lower halfline defined by a restricted formula. Let $\zeta=\operatorname{zup}(A)$. If $\forall x(x \in A \Leftrightarrow x \leq \zeta)$, then $\forall y(y \in B \Leftrightarrow \zeta<y)$. If $\forall x(x \in A \Leftrightarrow x<\zeta)$, then $\forall y(y \in B \Leftrightarrow \zeta \leq y)$. Finally, if $\forall x(x \in A \Leftrightarrow \forall z(z+e(\zeta)=\zeta \Rightarrow x<z))$, then $\forall y(y \in B \Leftrightarrow \exists z(z+e(\zeta)=\zeta \wedge z$ $\leq y))$. 
In the first case we call the upper halfline $B$ closed with minimum $\rho \equiv \min B$, in the second case we call the upper halfline open with infimum $\sigma \equiv \inf B$, and in the third case we call the upper halfline strongly open with weak infimum $\tau \equiv \operatorname{winf} B$. We may define greatest lower bounds for any set $B$ by defining winf $B \equiv$ winf $\underline{B}$ where $\underline{B} \equiv\{x \in S \mid \exists b \in B(b \leq x)\}$. Note that the complement of a closed lower halfline is open (if not empty), the complement of a open lower halfline is closed and the complement of a non-precise strongly open lower halfline is again strongly open. As a corollary to Theorem 3.11 we obtain the following criterion for upper halflines.

Corollary 3.13 Let $S$ be a complete solid and $B \subseteq S$ be an upper halfline defined by a restricted formula. The elements $\rho, \sigma$ and $\tau$ of Theorem 3.12 are unique and the cases 1 and 2, and the cases 1 and 3 are mutually exclusive. If $B$ is a precise lower halfline, it is strongly open if and only if it is closed. If $B$ is not a precise lower halfline, the properties of being open, closed or strongly open are mutually exclusive.

It is to be noted that also the case of the complete solid $S$ itself enters in the above classifications. Considered as a lower halfline it has a maximum in the form of the maximal magnitude $M$, while $M$ acts as a weak infimum, if $S$ is considered as an upper halfline.

Proposition 3.14 Let $S$ be a complete solid and $E$ be a set of magnitudes defined by a restricted formula. Then $\operatorname{zup} E$ and winf $E$ are magnitudes. In fact, zup $E$ is a maximum or a supremum and winf $E$ is a minimum or an infimum.

Proof Let $Z=\operatorname{zup} E$. If $Z$ is a maximum it is clearly a magnitude. Assume that $Z$ is a supremum. If $Z$ is zeroless, then $Z / 2<Z$. Then there must exist an element $f$ such that $f+f=f$ and $Z / 2<f<Z$, otherwise $Z / 2$ would already be an upper bound of $E$. Hence $Z<2 f=f$, a contradiction. Hence $Z$ is a magnitude.

We show that $Z$ cannot be a weak supremum. If such, $Z$ cannot be a magnitude, otherwise every element in $Z$ would be negative, in contradiction with the fact that $0 \leq Z$. Also $Z$ cannot be zeroless. Indeed, then $Z$ would be of the form $t+e(Z)$ with $t$ precise and $e(Z)<t$. Then $t / 2+e(Z)<Z$. Then there must exist an element $f$ such that $f+f=f$ and $t / 2+e(Z)<f<Z$, otherwise $t / 2+e(Z)$ would already be an upper bound of $E$. Hence $Z=t+e(Z)<2 f=f<Z$, a contradiction. We conclude that $Z$ is a maximum or a supremum.

The proof for winf $E$ is similar. 


\section{Limited numbers and infinitesimals}

Notation Let $S$ be a complete solid. With some abuse of language the winf of the magnitudes larger than 1 is noted $£$, ie $£ \equiv \operatorname{winf}\{e \in S \mid e+e=e \wedge 1<e\}$ and the zup of the magnitudes smaller than 1 is noted $\oslash$, ie $\oslash \equiv \operatorname{zup}\{e \in S \mid e+e=e \wedge e<1\}$.

Theorem 4.1 Within a complete solid the sets $\oslash$ and $£$ are magnitudes and satisfy

$$
0<\oslash<1<£<M .
$$

Proof Let $E=\{e \mid e+e=e \wedge e<1\}$. By Axiom 2.27 there exists $e$ such that $e+e=e$ and $0<e<M$. If $1<e$ then there is a precise element $p$ such that $1<e<p<M$. Then $0<1 / p<e / p<1$. If $e<1$ then there is a precise element $q$ such that $0<q<e$. Then $0<1<e / q<1 / q<M$. Hence there exists a magnitude between 0 and 1 and a magnitude between 1 and $M$. So $0<\oslash$ and $£<M$.

By Proposition 3.14, $\oslash$ is a magnitude. If $\oslash$ is a maximum, clearly $\oslash<1$. Assume $\oslash$ is a supremum. If $1<\oslash$, there must exist an element $e$ such that $e+e=e$ and $1<e<1$, a contradiction. We conclude that $\oslash<1$.

The proof that $£$ is a magnitude and $1<£$ is analogous, now using $F=\{e \mid e+e=$ $e \wedge 1<e\}$.

Corollary 4.2 There are no magnitudes between the magnitudes $\oslash$ and $£$.

Since $0<\oslash$ and $£<M$, by Axiom 2.29 and Lemma 3.7 there are precise elements $p$ and $q$ such that $0<p<\oslash$ and $£<q<M$.

Below we show that $\oslash$ and $£$ are idempotent magnitudes for multiplication, ie $\oslash \oslash=\oslash$ and $£ £=£$. Moreover $\oslash £=\oslash$ or $\oslash £=£$. Indeed, it follows from Axiom 2.19 that the product of two magnitudes $e$ and $f$ is a magnitude. In particular $\oslash £$ is a magnitude. By compatibility with the ordering,

$$
\oslash \leq \oslash 1 \leq \oslash £ \leq 1 £=£ .
$$

To decide whether $\oslash £=\oslash$ or $\oslash £=£$, we need Axiom 2.30. In fact $\oslash £=\oslash$, which will be shown in Section 5. Next lemma states some basic properties of $\oslash$ and $£$.

Lemma 4.3 Let $0<p$ be precise. Then

(1) $£<p$ if and only if $1 / p<\oslash$ 
(2) $\oslash<p$ if and only if $1 / p<£$

(3) If $p<\oslash$ then $\sqrt{p}<\oslash$

(4) If $£<p$ then $£<\sqrt{p}$

(5) If $\oslash<p<£$, then $\oslash<p^{2}<£$

(6) $\oslash=\sup \{p \mid e(p)=0 \wedge £<1 / p\}$ and $£=\inf \{1 / p \mid e(p)=0 \wedge p<\oslash\}$

Proof 1 . Assume $£<p$. Then $£ / p \leq 1$. Because $£ / p$ is a magnitude, we have $£ / p<1$, so $£ / p \leq \oslash$. Since $1 / p<£ / p$ we derive that $1 / p<\oslash$. Assume now that $1 / p<\oslash$. Then $1 \leq p \oslash$, in fact $1<p \oslash$, because $p \oslash$ is a magnitude. So $£ \leq p \oslash<p \cdot 1=p$. Hence $£<p$ if and only if $1 / p<\oslash$.

2. Directly from Lemma 4.3.1.

3. Suppose that $p<\oslash$ and $\oslash<\sqrt{p}$. Then $\oslash / \sqrt{p}<1$. Then $\oslash / \sqrt{p} \leq \oslash$. Hence $p<\oslash \leq \sqrt{p} \oslash$, so $\sqrt{p}<\oslash$, a contradiction. Hence $\sqrt{p}<\oslash$.

4. Suppose that $£<p$ and $\sqrt{p}<£$. Then $1<£ / \sqrt{p}$. Then $£ \leq £ / \sqrt{p}$. Hence $\sqrt{p} £ \leq £<p$, so $£<\sqrt{p}$, a contradiction. Hence $£<\sqrt{p}$.

5. This part is a direct consequence of Lemma 4.3.3 and 4.3.4.

6. Let $0<p$ be precise. Put $\oslash^{\prime}=\sup \{p \mid e(p)=0 \wedge £<1 / p\}$. Suppose that $\oslash<\oslash^{\prime}$. Then there exists a precise element $q$ such that $\oslash<q<\oslash^{\prime}$. Then $1 / q<£$ by Lemma 4.3.2, while $£<1 / q$ by definition of $\oslash^{\prime}$. Hence $\oslash^{\prime} \leq \oslash$. Suppose now that $\oslash^{\prime}<\oslash$. Then there exists a precise element $r$ such that $\oslash^{\prime}<r<\oslash$. Then $1 / r<£$ by definition of $\oslash^{\prime}$, while $£<1 / r$ by Lemma 4.3.1, a contradiction. Hence $\oslash=\oslash^{\prime}$. The second part is proved in an analogous way.

Theorem 4.4 One has:

(1) $\oslash \oslash=\oslash$

(2) $£ £=£$

Proof 1. Suppose $\oslash \oslash<\oslash$. Then there is a precise element $p$ such that $\oslash \oslash<p<\oslash$. By Lemma 4.3.3 one has $\sqrt{p}<\oslash$. Then $p=\sqrt{p} \sqrt{p}<\oslash \oslash$, a contradiction. Hence $\oslash \leq \oslash \oslash$. Because $\oslash<1$, also $\oslash \oslash \leq \oslash$. We conclude that $\oslash \oslash=\oslash$.

2. Suppose $£<£ £$. Then there is a precise element $p$ such that $£<p<£ £$. By Lemma 4.3 .4 one has $£<\sqrt{p}$. Then $£ £<\sqrt{p} \sqrt{p}=p$, a contradiction. Hence $£ £ \leq £$. Because $1<£$, also $£ \leq £ £$. We conclude that $£ £=£$. 


\section{Product of magnitudes}

Let $f$ and $g$ be two magnitudes. Though the product $f g$ is well-defined as a magnitude, the value of this magnitude is not determined. For example, formula (4) and Corollary 4.2 show that $\oslash £=\oslash$ or $\oslash £=£$ but do not decide which equality holds. We will see that Axiom 2.30 has as a consequence that $\oslash £=\oslash$. In fact this axiom together with Axiom 2.31 implies that the value of the product $f g$ is determined for all magnitudes $f$ and $g$. Axiom 2.30 gives the value of the product of a magnitude which is idempotent for multiplication with its so-called maximal ideal. Using an order argument, it will be shown that the axiom determines the value of the product of all magnitudes which are idempotent for multiplication. Axiom 2.31 states that every magnitude is a multiple of an idempotent magnitude which is shown to be unique. This enables to determine all products of magnitudes.

We recall first some definitions from Section 2.2.1.

Definition 5.1 A magnitude $I$ is called idempotent if $I I=I$.

Clearly 0 and $M$ are idempotent magnitudes and by Theorem 4.4, also $\oslash$ and $£$. Note that if $e$ and $f$ are idempotent then $e f$ is also idempotent, because efef $=e e f f=e f$.

Definition 5.2 Let $e$ and $I$ be magnitudes such that $1<I, I$ is idempotent and $e \leq I$. If for all precise positive $q$ such that $q<I$ it holds that $e q \leq e$ we say that $e$ is an ideal of $I$. An ideal $e$ of $I$ is said to be maximal if $e<I$ and for every ideal $f$ of $I$ such that $e \leq f \leq I$ one has $e=f$ or $f=I$.

Every idempotent magnitude $I$ such that $1<I$ possesses an ideal. Indeed, 0 is an ideal of $I$, for 0 is an idempotent magnitude and for all precise $q$ such that $q<I$ one has $0 q=0$. If $I<M$, then $I$ has nonzero ideals and the existence of a maximal ideal of $I$ will be a consequence of generalized Dedekind completeness.

Notation Unless otherwise said, we let $J$ be an idempotent magnitude such that $1<J<M$ and $I=\sup A$, where $A \equiv\{1 / \omega \mid \omega$ precise, $J<|\omega|\}$.

Theorem 5.3 The maximal ideal of $M$ is equal to 0 . If $1<J<M$, then $0<I \leq \oslash$ and $I$ is the maximal ideal of $J$.

The first part of the theorem follows from the fact that $x 0=0$ for all $x \in S$ such that $x<M$ (Dinis and Van den Berg [10, Proposition 3.5]), and $x M=M$ for all $x \in S$ such that $x \neq 0$. Observe that as a consequence $0 M=M$. To prove the remaining part we start with some preparatory lemmas. 
Lemma 5.4 Let $0<p$ be precise. Then

(1) If $p<I$ then $2 p<I$.

(2) If $p<I$ then $\sqrt{p}<I$.

(3) If $1 \leq p<J$ then $I<1 / p$.

Proof Assume that $p<I$. Then $p \in A$. Hence $J<1 / p$. Then $J<1 / 2 p$ because $J$ is a magnitude and $J<1 / \sqrt{p}$ because $J$ is idempotent. Hence $2 p<I$ and $\sqrt{p}<I$. This proves Lemma 5.4.1 and 5.4.2. Lemma 5.4.3 follows directly from the definition of $I$.

Lemma $5.5 I<1$ is an idempotent magnitude.

Proof The fact that $I<1$ follows from Lemma 5.4.3. It follows from Lemma 5.4.1 that $I \leq I+I$. Suppose that $I<I+I$. Then there exists a precise element $p$ such that $I<p<I+I$. Then $p=p / 2+p / 2<I$ by Lemma 5.4.1, a contradiction. Hence $I$ is a magnitude. To show that $I$ is idempotent, observe that $I I \leq I$ because $I<1$. Suppose $I I<I$. Then there exists a precise element $p$ such that $I I<p<I$. Then $p=\sqrt{p} \sqrt{p}<I$ by Lemma 5.4.2, a contradiction. Hence $I$ is idempotent.

Lemma 5.6 Let $r, p$ be precise and such that $1<p<J$ and $r<I$. Then $r p<I$.

Proof By the definition of $I$ one has $J<1 / r$. Suppose that $1 /(r p)=(1 / r) / p<J$. Then $1 / r<p J<J J=J$, a contradiction. Hence $J<1 /(r p)$ and $r p<I$, by the definition of $I$.

Lemma $5.7 \quad I$ is an ideal of $J$.

Proof It is enough to prove that $I p \leq I$ for all precise $p$ such that $1<p<J$. By Lemma 5.6, Ip $=\sup \{r \mid e(r)=0 \wedge r<I\} \cdot p=\sup \{p r \mid e(r)=0 \wedge r<I\} \leq I$.

Lemma 5.8 Let $K$ be an ideal of $J$. Then $K \leq I$ or $K=J$.

Proof Assume that $K$ is an ideal of $J$ such that $I<K$. Then there exists a precise $p$ such that $I<p<K$. Then $1 / p<J$, so $1=p \cdot 1 / p<K \cdot 1 / p \leq K$. Suppose $K<J$. Then there exists a precise $q$ such that $K<q<J$. Then $q=1 \cdot q<K q \leq K$, a contradiction. Hence $K=J$ or $K \leq I$. 
Proof of Theorem 5.3 Lemma 5.7 states that $I$ is an ideal of $J$. By Lemma 5.8, every ideal of $J$ which is different from $J$ is less than or equal to $I$. Hence $I$ is the maximal ideal of $J$. The fact that $I \leq \oslash$ follows from Lemma 5.5.

We will now determine the value of the product of two magnitudes. Theorem 5.9 below states that the product of idempotents is equal to one of the factors and Proposition 5.10 deals with the special case of the product of $\oslash$ and $£$. Proposition 5.11 states that, for non-zero magnitudes, the idempotent magnitude given by Axiom 2.31 is unique. Then the value of the product of magnitudes follows by applying Axiom 2.31 and Theorem 5.9 and comes in the form of a linearization, otherwise said, the product of two magnitudes is a multiple of one of them.

Theorem 5.9 Let $e$ and $f$ be idempotent magnitudes with $e \leq f$. If $f<1$, then $e f=e$. If $1<f$ we let $I$ be the maximal ideal of $f$. Then ef $=e$ if $e \leq I$, and $e f=f$ if $I<e$.

Proof If $e=f$ the property is obvious. If $e<f<1$, we have $e f=e$, for $e=e e \leq e f \leq e \cdot 1=e$. If $1<e<f$, we have $e f=f$, for $f=1 \cdot f \leq e f \leq f f=f$. Finally, assume that $e<1<f$. Assume that $e \leq I$. By the above we have $e I=e$. Then by Axiom 2.30 one has $e f=e I f=e I=e$. Assume that $I<e$. Note that $e f$ is an ideal of $f$, for $e f q \leq e f f=e f$ for all precise $q<f$. Also $I<e=e e \leq e f \leq f$. Hence $e f=f$.

Notice that by commutativity the product ef is also determined if $f<e$.

Proposition 5.10 The magnitude $\oslash$ is the maximal ideal of $£$. As a consequence $\oslash £=\oslash$.

Proof By Theorem 4.4.2 the magnitude $£$ is idempotent. It follows from Lemma 4.3.6 and Theorem 5.3 that $\oslash$ is the maximal ideal of $£$. Then $\oslash £=\oslash$ by Theorem 5.9 .

Proposition 5.11 Let $e$ be a nonzero magnitude and $I$ be an idempotent magnitude such that $e=p I$ for some precise element $p$. Then $I$ is unique.

Proof Let $e$ be a magnitude. Suppose that there exist idempotent magnitudes $I, J \in S$ and precise elements $p$ and $q$ such that $e=p I$ and $e=q J$. Then $p I=q J$. Because $e \neq 0$ the elements $p$ and $q$ are non-zero. Then, noting that $I$ and $J$ are idempotent,

$$
I J=I\left(\frac{p}{q} I\right)=\frac{p}{q} I=J
$$


and

$$
I J=\left(\frac{q}{p} J\right) J=\frac{q}{p} J=I .
$$

We conclude that $I=J$.

Theorem 5.12 Let $e$ and $f$ be magnitudes. Then the value of $e f$ is uniquely determined. Moreover, there exists a positive precise $p$ such that $e f=p f$ or a positive precise $q$ such that ef $=q e$.

Proof Clearly ef $=0$ if $e=0$ or $f=0$, and we may take $q=1$ or $p=1$ respectively. Let $e$ and $f$ be non-zero magnitudes. By Axiom 2.31 there exist idempotent $I$ and $J$ and precise $p$ and $q$ such that $e=p I$ and $f=q J$; dealing with magnitudes they may be supposed positive. Then $e f=p q I J$. Now $I J=I$ or $I J=J$ by Theorem 5.9 and the value of the product $e f$ is uniquely determined by Proposition 5.11. Hence $e f=p f$ or ef $=q e$.

In the last part of this section we verify that the value of the product obtained from Axiom 2.30 is consistent with the ordering (Theorem 5.14) and the notion of supremum of Axiom 2.32 (Theorem 5.15).

Lemma 5.13 Let $J>1$ be an idempotent magnitude and $I$ be the maximal ideal of $J$. Let $p$ be a precise element.

(1) If $p<I$ then $p J<I$.

(2) If $I<p$ then $J \leq p J$. Moreover, $p J=J$ if and only if $I<p<J$.

(3) If $J<p$ then $J<p I$.

(4) If $p<J$ then $p I \leq I$. Moreover, $p I=I$ if and only if $I<p<J$.

(5) There is no precise element $p$ such that $p I=J$.

Proof 1. Assume that $p<I$. Then $\sqrt{p}<I$ by Lemma 5.4.2. Then $J<1 / \sqrt{p}$. Hence

$$
p J<p \frac{1}{\sqrt{p}}=\sqrt{p}<I .
$$

2. Assume that $I<p$. Then $I<p^{2}$ by idempotency. If $1 \leq p$, clearly $J \leq p J$. If $p<1$, then $p<J$. Suppose that $p J<J$. Because $p J$ is an ideal of $J$, one has $p J \leq I<p^{2}$. Then $J<p$, a contradiction. Hence $J \leq p J$. Assume that $I<p<J$. 
Then $p J \leq J^{2}=J$. Hence $p J=J$. For $J<p$ we have $J<p<p J$. Hence $p J=J$ if and only if $I<p<J$.

3. Suppose that $J<p$. Then $J<\sqrt{p}$ by idempotency. Then $1 / \sqrt{p}<I$, hence

$$
J<\sqrt{p}=p \frac{1}{\sqrt{p}}<p I .
$$

4. Because $I$ is an ideal of $J$ we have $p I \leq I$. Assume that $I<p<J$. Then $I=I^{2} \leq p I$. Hence $p I=I$. By Lemma 5.13.1 one has $p I \leq p J<I$ for $p<I$. Hence $p I=I$ if and only if $I<p<J$.

5. Directly from Lemma 5.13.3 and 5.13.4.

Theorem 5.14 Let $J>1$ be an idempotent magnitude and $I$ be the maximal ideal of $J$. Let $p, q>0$ be precise.

(1) If $p<I<q$ then $p J<I J<q J$.

(2) If $p<J<q$ then I $\leq I J<I q$.

Proof 1. By Lemmas 5.13.1 and 5.13.2, pJ $<I=I J<J \leq q J$.

2. By Lemmas 5.13.4 and 5.13.3, Ip $\leq I=I J<J<I q$.

Theorem 5.15 Let I, $J$ be idempotent magnitudes such that $1<J$ and $I$ is the maximal ideal of $J$. Then:

(1) $I=I J=\sup \{p J|e(p)=0| p \mid,<I\}=\max \{I q|e(q)=0| q \mid,<J\}$

(2) $J=\inf \{p I \mid e(p)=0, J<p\}=\min \{q J \mid e(q)=0, I<q\}$

Proof 1. By Lemma 5.13.1, if $p<I$ then $p J<I$. Also $1<J$. Hence

$$
I=\sup \{p|e(p)=0,| p \mid<I\} \leq \sup \{p J|e(p)=0,| p \mid<I\} \leq I .
$$

Hence $I J=\sup \{p J|e(p)=0| p \mid,<I\}$. Also $I q \leq I J$ for all precise $q$ with $|q|<J$. By Lemma 5.13.4 one has $I q=I=I J$ for all precise $q$ with $I<|q|<J$. Hence $I J=\max \{I q|e(q)=0| q \mid,<J\}$.

2. By Lemma 5.13.3 we have $J \leq \inf \{p I \mid J<p\}$. In order to show that also $\inf \{p I \mid J<p\} \leq J$, suppose towards a contradiction that $J<\inf \{p I \mid J<p\}$. Then there exists a precise element $q$ such that $J<q<\inf \{p I \mid J<p\}$. Since $J<q$, one has inf $\{p I \mid J<p\} \leq q I$. Then $q<q I$, which implies that $1<I$, a contradiction. 
One concludes that inf $\{p I \mid J<p\}=J$. By Lemma 5.13.3, inf $\{p I \mid J<p\}$ is not a minimum. By Proposition 3.14, inf $\{p I \mid J<p\}$ is an infimum.

By Lemma 5.13.2 one has $J \leq p J$ for $I<p$, and in particular $p J=J$ for all $p$ with $I<p<J$. Hence $J=\min \{p J \mid v I<p\}$.

Theorem 5.15 also states that we may obtain $I J=I$ by approximation from below, but not by approximation from above. This shows that completion arguments are not enough to determine the product of magnitudes.

\section{On consistency}

In this section we show that Axioms 2.1-2.35 are consistent by constructing a model extending a particular nonstandard model of the real numbers. Indeed, we take a sufficiently saturated nonstandard model ${ }^{*} \mathbb{R}$ of the real numbers which is elementary equivalent to $\mathbb{R}$. Within this model we consider cosets with respect to convex subgroups which are definable by $\Sigma_{1}$ or $\Pi_{1}$ formulas. The resulting structure will be called $\mathcal{E}$. As we will see, all the axioms presented in Section 2 are valid in $\mathcal{E}$.

In a previous article [11] we made an interpretation of most of the algebraic axioms using the language of Nelson's Internal Set Theory [23]. More precisely we used an adapted version, formulated by Kanovei-Reeken [17], which permits to include external sets. As it turns out, in this approach the collection of magnitudes is a proper class. To avoid foundational problems, which may appear when we apply, say, asymptotics with parameters or defining subclasses, we will consider here a semantic approach.

The axioms for a solid, ie Axioms 2.1-2.29, extend the axioms originally presented in [11] and were shown to be consistent in Dinis and Van den Berg [12] by the construction of a direct model in the language of $Z F C$. This was given in the form of a set of cosets of a non-Archimedean field. Allowing for definable classes, it was shown in [11] that the external numbers of Koudjeti [19] and Koudjeti and Van den Berg [20] satisfy the axioms for addition and for multiplication, together with a modified form of the distributivity axiom; this modified form was shown to be equivalent to Axiom 2.22 in Dinis and Van den Berg [10]. The remaining algebraic axioms deal with multiplication of magnitudes, and are in fact taken from calculation rules of the external numbers observed in [19] and [20]. 


\subsection{Construction of the solid $\mathcal{E}$}

Let $Z_{n}=\bigcup_{k \leq n} P^{k}(\mathbb{R})$ and $Z=\bigcup_{n \in \mathbb{N}} Z_{n}$ be the superstructure of Robinson and Zakon [26] (see also Stroyan and Luxemburg [28] and Goldblatt [13]). Let ${ }^{*} Z$ be an adequate ultralimit [23] of $Z$. If we interpret the elements of $Z$ as standard, we will see that bounded versions of the axioms $I, S$, and $T$ of Nelson, as well as his Reduction Algorithm hold in this structure. In particular, the Saturation Principle [25, Theorem 5] holds. In the context of the superstructure this implies that if $X \in Z$ and $s: X \rightarrow{ }^{*} \mathbb{R}$, then $s$ has always an internal extension $\widetilde{s}:{ }^{*} X \rightarrow{ }^{*} \mathbb{R}$.

Definition 6.1 We denote by $\mathcal{N}$ the set consisting of ${ }^{*} \mathbb{R}$ and of all convex subgroups of $* \mathbb{R}$ of the form $\bigcup_{x \in X}\left[-s_{x}, s_{x}\right]$ or $\bigcap_{x \in X}\left[-s_{x}, s_{x}\right]$, where $X \in Z$ and $s: X \rightarrow{ }^{*} \mathbb{R}$. We call an element of $\mathcal{N}$ a neutrix.

Without restriction of generality we may suppose that $X$ is ordered and $s$ is increasing in the case of unions and decreasing in the case of intersections.

Definition 6.2 Let $A, B$ be neutrices. With some abuse of language we call the set $\left\{a^{*}+b \mid a \in A \wedge b \in B\right\}$ the Minkowski sum of $A$ and $B$ and the set $\left\{a^{*} \cdot b \mid a \in\right.$ $A \wedge b \in B\}$ the Minkowski product of $A$ and $B$. Usually we simply write $A+B$ instead of $A^{*}+B$ and $A \cdot B$ instead of $A^{*} \cdot B$.

Definition 6.3 We define $\mathcal{E}=\left\{a+A \mid a \in{ }^{*} \mathbb{R} \wedge A \in \mathcal{N}\right\}$. We call an element of $\mathcal{E}$ an external number.

If $\alpha=a+A$ is an external number, it is tacitly understood that $a \in{ }^{*} \mathbb{R}$ and $A \in \mathcal{N}$. We write $N(\alpha)$ instead of $A$ and call it the neutrix part of $\alpha$. This functional notation will be justified below.

Proposition 6.4 Let $\alpha=a+A$ be an external number.

(1) Let $y \in \alpha$. Then $\alpha=y+A$.

(2) Let $\alpha=b+B$ with $b \in{ }^{*} \mathbb{R}$ and $B \in \mathcal{N}$. Then $A=B$.

Proof 1. We have that $y-a \in \alpha-\alpha=A$. Then $y+A=y-a+a+A \subseteq a+A+A=\alpha$. On the other hand, $\alpha=a+A=a-y+y+A \subseteq y+A+A=y+A$. Hence $\alpha=y+A$.

2. We have $A=\alpha-\alpha=B$.

Corollary 6.5 The neutrix part is a well-defined function from $\mathcal{E}$ to $\mathcal{N}$. 
Obviously $\alpha=y+A$ for any element $y \in \alpha$. The neutrix part of a given external number is unique and functional. Next definition extends the Minkowski sum and product of Definition 6.2 to external numbers. It is easy to see that the definition does not depend on the choice of representatives.

Definition 6.6 Let $\alpha=a+A$ and $\beta=b+B$ be two external numbers, the sum and product of $\alpha$ and $\beta$ are defined as follows:

$$
\begin{aligned}
\alpha+\beta & =a+b+A+B \\
\alpha \cdot \beta & =a b+a B+b A+A B
\end{aligned}
$$

Let $A, B$ be neutrices and $\left(s_{x}\right)_{x \in X},\left(t_{y}\right)_{y \in Y}$ be families of elements of * $\mathbb{R}$, with $X, Y \in Z$. Because we are only considering convex subgroups of $* \mathbb{R}$ of the form $\bigcup_{x \in X}\left[-s_{X}, s_{x}\right]$ or $\bigcap_{x \in X}\left[-s_{x}, s_{x}\right]$, we need to show that the sum and product operations do not increase the complexity. With addition complexity does not increase because one always has $A+B=A$ or $A+B=B$. We now consider multiplication. There is clearly no increase in complexity if both $A$ and $B$ are unions, or are intersections. Indeed

$$
\bigcup_{x \in X}\left[-s_{x}, s_{x}\right] \cdot \bigcup_{y \in Y}\left[-t_{y}, t_{y}\right]=\bigcup_{(x, y) \in X \times Y}\left[-s_{x}, s_{x}\right]\left[-t_{y}, t_{y}\right],
$$

and

$$
\bigcap_{x \in X}\left[-s_{x}, s_{x}\right] \cdot \bigcap_{y \in Y}\left[-t_{y}, t_{y}\right]=\bigcap_{(x, y) \in X \times Y}\left[-s_{x}, s_{x}\right]\left[-t_{y}, t_{y}\right] .
$$

In the following proposition we show that there is also no increase in complexity in the case where $A$ is of the form $\bigcup_{x \in X}\left[-s_{x}, s_{x}\right]$ and $B$ is of the form $\bigcap_{y \in Y}\left[-t_{y}, t_{y}\right]$.

Proposition 6.7 Let $X, Y \in Z$. Let $A=\bigcup_{x \in X}\left[-s_{x}, s_{x}\right]$ and $B=\bigcap_{y \in Y}\left[-t_{y}, t_{y}\right]$ be neutrices where $\left(s_{x}\right)_{x \in X},\left(t_{y}\right)_{y \in Y}$ are families of elements of ${ }^{*} \mathbb{R}$. Then $A B=$ $\bigcup_{w \in W}\left[-u_{w}, u_{w}\right]$ or $A B=\bigcap_{w \in W}\left[-u_{w}, u_{w}\right]$, where $\left(u_{w}\right)_{w \in W}$ is a family of elements of ${ }^{*} \mathbb{R}$ with $W=X$ or $W=Y$.

Proof To $A B$ we associate the halfline $C=]-\infty, A B]$. This halfline is of the form $\left.\left.\bigcup_{x \in X}\right]-\infty, u_{x}\right]$ where $u: X \rightarrow{ }^{*} \mathbb{R}$ is internal or of the form $\left.\left.\bigcap_{y \in Y}\right]-\infty, u_{y}\right]$ where $u: Y \rightarrow{ }^{*} \mathbb{R}$ is internal (see Van den Berg [2, Theorem 4.33]). The proposition is a direct consequence of this fact.

Definition 6.8 Let ${ }^{*} \leq$ be the order relation on ${ }^{*} \mathbb{R}$. Given $\alpha, \beta \in \mathcal{E}$, we write, with some abuse of language, $\alpha \leq \beta$ if and only if

$$
(\forall x \in \alpha)(\exists y \in \beta)\left(x^{*} \leq y\right) .
$$


Let $\alpha \in \mathcal{E}$. Let $Q_{\alpha}=\{x \in \mathcal{E} \mid x \leq \alpha\}$. Then $\alpha \leq \beta$ if and only if $Q_{\alpha} \subseteq Q_{\beta}$.

Note that if $\alpha \cap \beta=\emptyset$, formula (5) is equivalent to $(\forall x \in \alpha)(\forall y \in \beta)\left(x^{*}<y\right)$. Lemma 6.9 shows that two external numbers are always either disjoint or one contains the other (see also Koudjeti [19, Proposition 3.2.15]).

Lemma 6.9 Let $\alpha$ and $\beta$ be two external numbers. Then

$$
\alpha \cap \beta=\emptyset \vee \alpha \subseteq \beta \vee \beta \subseteq \alpha .
$$

Proof Suppose that $\alpha \cap \beta \neq \emptyset$. Then there is $x \in{ }^{*} \mathbb{R}$ such that $x \in \alpha$ and $x \in \beta$. Then we may write $\alpha=x+A$ and $\beta=x+B$. Hence $\beta \subseteq \alpha$ if $\max (A, B)=A$, and $\alpha \subseteq \beta$ if $\max (A, B)=B$.

\subsection{The solid $\mathcal{E}$ as a model for the axioms}

In this section we show that the external numbers of the previous section are a model for the axioms. We will work progressively, and start with the algebraic axioms of a solid.

Theorem 6.10 The structure $(\mathcal{E},+, \cdot, \leq)$ satisfies Axioms 2.1-2.29.

In order to prove the theorem, we verify first that the axioms for addition and the axioms for multiplication are satisfied. For the order axioms we will need to recapitulate in a modified way some results from Koudjeti [19] and Koudjeti and Van den Berg [20]. Then we show that the axioms relating addition and multiplication are satisfied and finally we show that the existence axioms are verified.

Proposition 6.11 The structure $(\mathcal{E},+, \cdot, \leq)$ satisfies Axioms 2.1-2.10.

Proof The proof is essentially the same as the proof given in Dinis and Van den Berg [11, Theorem 4.10].

Proposition 6.12 The structure $(\mathcal{E},+, \cdot, \leq)$ satisfies Axioms 2.11-2.18.

Before proving the proposition, we recall a lemma from [20].

Lemma 6.13 Let $A$ be a neutrix and let $\beta$ and $\gamma$ be external numbers such that $\beta \leq \gamma$. Then $A \beta \subseteq A \gamma$. 
Proof Assume that $\beta \leq \gamma$. Let $x \in \beta$ and $a \in A$. There exists $y \in \gamma$ such that $x \leq y$. Then $|a| x \leq|a| y \in A \gamma$. Hence $A \beta \subseteq A \gamma$.

Proof of Proposition 6.12 Working with halflines, it is immediate to see that the order relation is reflexive, transitive, antisymmetric and total. Then Axioms 2.11-2.14 are satisfied.

In order to show that Axiom 2.15 is satisfied assume that $\alpha \leq \beta$. Let $a \in \alpha$ and $c \in \gamma$. There exists $b \in \beta$ such that $a \leq b$. Hence $a+c \leq b+c \in \beta+\gamma$ and one concludes that $\alpha+\gamma \leq \beta+\gamma$. As regards to Axiom 2.16, assume that $\alpha+N(\beta)=N(\beta)$, ie $a+A+B=B$. Then $a+A \subseteq B$. Hence $\alpha \leq N(\beta)$. We now turn to Axiom 2.17. Assume that $N(\alpha) \leq \alpha$ and $\beta \leq \gamma$. If $\alpha=A$, then $A \beta \subseteq A \gamma$ by Lemma 6.13, so $A \beta \leq A \gamma$. If $A<\alpha$ and $x \in \alpha$ then $0<x$. Let $y \in \beta$. Because $\beta \leq \gamma$ there exists $z \in \gamma$ such that $y \leq z$. Then $x y \leq x z$. Hence $\alpha \beta \leq \alpha \gamma$. Finally, to prove that Axiom 2.18 holds suppose that $N(\beta) \leq \beta$ and $\beta \leq \gamma$. Let $z \in A \beta$. We may assume that $z$ is positive. Then there exist $a^{\prime} \in A, b^{\prime} \in \beta$ such that $z=a^{\prime} b^{\prime}$, moreover $a^{\prime}$ may be supposed positive. Because $b^{\prime} \in \beta$ there is $c^{\prime} \in \gamma$ such that $b^{\prime} \leq c^{\prime}$. Then $a^{\prime} b^{\prime} \leq a^{\prime} c^{\prime} \in A \gamma$. Hence $A \beta \subseteq A \gamma$, which implies that $A \beta \leq A \gamma$.

We turn now to the axioms which relate addition and multiplication. It was shown in Dinis and Van den Berg [11] that distributivity holds for external numbers under certain conditions. In Dinis and Van den Berg [10] equivalence was shown with Axiom 2.22. Here we give a direct proof that Axiom 2.22 holds in $\mathcal{E}$. We recall that for external numbers, being convex sets, subdistributivity always holds in the sense of inclusion, ie

$$
\alpha(\beta+\gamma) \subseteq \alpha \beta+\alpha \gamma .
$$

Theorem 6.14 Let $\alpha=a+A, \beta$ and $\gamma$ be external numbers. Then

$$
\alpha \beta+\alpha \gamma=\alpha(\beta+\gamma)+A \beta+A \gamma \text {. }
$$

Proof It is easy to see [11] that distributivity holds in the case that $\alpha=a$ is precise and that $(a+A)(\beta+\gamma)=a(\beta+\gamma)+A(\beta+\gamma)$. Then

$$
\begin{aligned}
\alpha(\beta+\gamma)+A \beta+A \gamma & =(a+A)(\beta+\gamma)+A \beta+A \gamma \\
& =a(\beta+\gamma)+A(\beta+\gamma)+A \beta+A \gamma \\
& =a \beta+a \gamma+A(\beta+\gamma)+A \beta+A \gamma .
\end{aligned}
$$

By formula (6) and because $A \beta, A \gamma$ and $A(\beta+\gamma)$ are neutrices, one has that $A \beta+A \gamma+A(\beta+\gamma)=A \beta+A \gamma$.

Hence $\alpha(\beta+\gamma)+A \beta+A \gamma=a \beta+a \gamma+A \beta+A \gamma=\alpha \beta+\alpha \gamma$. 
Proposition 6.15 The structure $(\mathcal{E},+, \cdot, \leq)$ satisfies Axioms 2.19-2.23.

Proof For Axioms 2.20, 2.21 and 2.23 we refer to [11, Propositions 4.15 and 4.17]. Axiom 2.22 holds by Theorem 6.14. We still must show that Axiom 2.19 is satisfied. Let $A \in \mathcal{N}$ and $\beta=b+B \in \mathcal{E}$. One has

$$
A(b+B)=b A+A B=\max (b A, A B) .
$$

Clearly $b A \in \mathcal{N}$, and $A B \in \mathcal{N}$ follows from Proposition 6.7.

We consider now the group of axioms on existence. We prove first the existence of representatives of the special elements $m, u$ and $M$.

Proposition 6.16 The structure $(\mathcal{E},+, \cdot, \leq)$ satisfies Axioms 2.24-2.26.

Proof The proposition follows by putting $m=0, M={ }^{*} \mathbb{R}$.

Axiom 2.27 states the existence of magnitudes between the smallest element $m$ and the largest element $M$. With Generalized Dedekind completeness we defined a largest magnitude $\oslash$ such that $0<\oslash<1$ and a smallest magnitude $£$ such that $1<£<M$. We will interpret $\oslash$ and $£$ in the following way, where we identify $\mathbb{N}$ with the standard integers of $* \mathbb{R}$.

Definition 6.17 We define $\left.\Lambda=\bigcup_{n \in \mathbb{N}}{ }^{*}\right]-n, n\left[\right.$ and $\left.\Theta=\bigcap_{n \in \mathbb{N}}{ }^{*}\right]-1 / n, 1 / n[$.

Theorem 6.18 The external sets $\Theta$ and $\Lambda$ are neutrices. One has $0<\Theta<1<\Lambda<$ $* \mathbb{R}$. The interpretation of $\oslash$ is $\Theta$ and the interpretation of $£$ is $\Lambda$.

Proof Clearly $\Theta$ and $\Lambda$ are neutrices. Because $* \mathbb{R}$ is a superstructure of $\mathbb{R}$ there exists an infinitely large element $\nu$ in ${ }^{*} \mathbb{R}$. Clearly $\nu \notin \Lambda$ hence $\Lambda \neq{ }^{*} \mathbb{R}$. Also $0 \neq 1 / \nu \in \Theta$. Obviously $\Theta<1<\Lambda$, because $1 \in \Lambda$ and $1 \notin \Theta$. Let $L, I$ be the interpretations of $£$ and $\oslash$ respectively. Then $L, I$ must be neutrices. There does not exist a proper convex subset of $\mathbb{N}$ closed under addition so there does not exist a neutrix $A$ such that $1<A<\Lambda$. Also, there does not exist a neutrix $B$ such that $\Theta<B<1$. Hence $\Lambda \subseteq L$ and $I \subseteq \Theta$. Because $\Lambda+\Lambda=\Lambda$ and $1<\Lambda$, by the definition of $£$ one has $L \subseteq \Lambda$. Also, because $\Theta+\Theta=\Theta$ and $\Theta<1$, by the definition of $\oslash$ one has $\Theta \subseteq I$. Hence $\Lambda=L$ and $\Theta=I$.

Proposition 6.19 The structure $(\mathcal{E},+, \cdot, \leq)$ satisfies Axioms 2.27-2.29. 
Proof By Theorem 6.18, Axiom 2.27 holds. Axiom 2.28 is trivially satisfied. Finally we turn to Axiom 2.29. Let $A, B \in \mathcal{N}$ be such that $A \neq B$. We may assume without loss of generality $A \varsubsetneqq B$. Then there is a nonstandard real number $b$ such that $b \in B$ and $b \notin A$. Furthermore, $b$ may be supposed positive. We show that $A<b<B$. Indeed, because $B$ is a group and $b$ is positive one has $b<2 b \in B$. Hence $b<B$. Suppose that $b \leq A$. Then there exists $a \in A$ such that $b \leq a$. Because $0 \in A$ and $a \in A$, by convexity $b \in A$, a contradiction. Hence $A<b$.

Proof of Theorem 6.10 The theorem follows by combining Proposition 6.11, Proposition 6.12, Proposition 6.15, Proposition 6.16 and Proposition 6.19.

The set $\mathcal{E}$ is not characterized by Axioms 2.1-2.29, for as showed in Dinis and Van den Berg [12] the set of all cosets with respect to all convex subgroups for addition of a non-Archimedean field is a model for these axioms. As we will see in the next section all the algebraic axioms, ie Axioms 2.1-2.29 together with the axioms 2.30 and 2.31, are still not sufficient for such a characterization.

We will now prove that the Generalized Completeness Axiom 2.32 holds in $\mathcal{E}$. We deal with this axiom before the axioms on multiplication of magnitudes, because Generalized Completeness is needed to prove the existence of the maximal ideals of Axiom 2.30.

In Van den Berg [2, Theorem 4.34, Corollary 4.35] (see also Diener and Diener [8, page 155]) a normal form for convex subsets of real numbers is stated. In the case of a (external) lower halfline this normal form indicates that its upper boundary is well-defined, in the form of a unique external number. The proof relies, in an essential way, on Nelson's Reduction Algorithm and on the Saturation Principle.

Let $Z$ be the superstructure defined in the previous section. In order to prove that the axiom on generalized completeness holds we interpret formulas from the language $\{+, \cdot, \leq\}$ in the adequate ultralimit ${ }^{*} Z$ and show that a bounded version of the Reduction Algorithm as well as the Saturation Principle hold in this structure.

Definition 6.20 Let $k$ be a natural number. Let $\Phi\left(x_{1}, \ldots, x_{k}\right)$ be a formula of $Z F C$ with free variables $x_{1}, \ldots, x_{k}$. The formula $\Phi$ is called bounded (relatively to $Z \cup{ }^{*} Z$ ) if all quantifications take the form $\forall x \in X$ or $\exists x \in X$, with $X \in Z$ or $X \in{ }^{*} Z$.

Definition 6.21 Let $k$ be a natural number. A bounded formula $\Phi\left(x_{1}, \ldots, x_{k}\right)$ is called internal (with some abuse of language) if all its quantifications take the form $\forall x \in X$ or $\exists x \in X$, with $X \in{ }^{*} Z$. 
Let $\Phi\left(x_{1}, \ldots, x_{k}\right)$ be a restricted formula of the language $\{+, \cdot, \leq\}$, this means that quantifications are only with respect to precise elements, see Subsection 2.2. We will interpret $\Phi$ by a formula $\bar{\Phi}$ in the structure $\mathcal{E}$ by induction on the complexity of the formula, and show that $\bar{\Phi}$ is bounded. Observe that a term $t\left(x_{1}, \ldots, x_{k}\right)$ is the result of a finite number of additions and multiplications of the variables $x_{1}, \ldots, x_{k}$. Each variable $x_{i}$ with $1 \leq i \leq k$ is interpreted by an element, say, $\alpha_{i}$ of $\mathcal{E}$; in particular, if $x_{i}$ is precise, then $\alpha_{i} \in{ }^{*} \mathbb{R}$. Then the interpretation $\bar{t}\left(\alpha_{1}, \ldots, \alpha_{k}\right)$ of $t$ is the result of a finite number of additions and multiplications of the elements $\alpha_{1}, \ldots, \alpha_{k}$. An atomic formula is of the form $t\left(x_{1}, \ldots, x_{k}\right) \leq s\left(y_{1}, \ldots, y_{m}\right)$, where $m$ is a natural number and $s$ is a term with variables $\left(y_{1}, \ldots, y_{m}\right)$. Then its interpretation is of the form

$$
\bar{t}\left(\alpha_{1}, \ldots, \alpha_{k}\right) \leq \bar{s}\left(\beta_{1}, \ldots, \beta_{m}\right),
$$

with $\beta_{1}, \ldots, \beta_{m} \in \mathcal{E}$. It follows from Definition 6.1 that the $\alpha_{i}$ and $\beta_{j}$ are either unions or intersections of families of intervals in $* \mathbb{R}$ indexed by elements of sets which are elements of $Z$. Hence the inequality (7) is expressed by a bounded formula.

Clearly the negation of a bounded formula is a bounded formula, and the conjunction of bounded formulas is a bounded formula. Since quantifiers in restricted formulas of the language $\{+, \cdot, \leq\}$ range over precise elements, quantifiers in their interpretations range over ${ }^{*} \mathbb{R}$, hence yield bounded formulas.

We conclude that the interpretation $\bar{\Phi}$ of $\Phi$ is bounded.

We show now that Nelson's Reduction Algorithm, properly adapted, transforms a bounded formula into a bounded formula of the form $\forall x \in X \exists y \in Y I(x, y)$, with $X, Y \in Z$ and $I(x, y)$ internal. Nelson's Reduction Algorithm uses three principles Transfer $(T)$, Idealization $(I)$ and modified Standardization $\left(S^{\prime}\right)$.

By Nelson [23] the Transfer Axiom and the Idealization Axiom of IST, when relativized to ${ }^{*} Z$, hold in ${ }^{*} Z$ indeed. In our context the modified Standardization Axiom takes the following form. Let $\Phi(x, y)$ be a bounded formula, this means that all quantifiers and parameters range over some $Z_{n}$. Let $m, n \in \mathbb{N}$ and $X, Y$ such that $X \subseteq Z_{m}$ and $Y \subseteq Z_{n}$. Assume that $\forall x \in Z_{m} \exists y \in Z_{n} \Phi(x, y)$. Then there must exist a function $\tilde{y} \in Z$ such that $\forall x \in X \Phi(x, \tilde{y}(x))$. This is true because if $\Phi$ is a formula of $Z F C$, by the Axiom of Choice there exists $\tilde{y}: X \rightarrow Y$ such that $\forall x \in X \Phi(x, \tilde{y}(x))$. Clearly $\tilde{y} \in Z$. So $\left(S^{\prime}\right)$ also holds in $Z$.

The bounded versions of the principles $I, S, T$ stated above hold in $Z^{*}$, ie applying any of these principles to a bounded formula results in a bounded formula. By the reasoning in the paragraph above this is clearly true for $\left(S^{\prime}\right)$. We verify the property also for $(T)$ and $(I)$. Let in the formulas below $\Phi$ always be a bounded formula. Then $(T)$ becomes

$$
\forall y \in Y\left(\forall x \in X \Phi(x, y) \leftrightarrow \forall x \in{ }^{*} X \Phi(x, y)\right),
$$


where $X, Y \in Z$ and $\Phi$ internal. Also ( $I$ ) becomes

$$
\begin{gathered}
\forall w \in{ }^{*} W\left(\forall v \in P_{\text {fin }}(X) \exists y \in{ }^{*} Y \forall x \in v \Phi(x, y, w)\right. \\
\left.\leftrightarrow \exists y \in{ }^{*} Y \forall x \in{ }^{*} X \Phi(x, y, w)\right),
\end{gathered}
$$

where $X, Y, W \in Z, \Phi$ internal and $P_{f i n}(X)$ is the set of all finite subsets of $X$. Note that $P_{\text {fin }}(X) \in Z$.

So we have the following theorem.

Theorem 6.22 Every bounded formula $\Phi$ is equivalent to a bounded formula of the form $\forall x \in X \exists y \in Y I(x, y)$, with $X, Y \in Z$ and $I(x, y)$ internal.

Because the Saturation Principle is true in bounded $I S T$ it also holds in ${ }^{*} Z$. We may now apply Van den Berg [2, Theorem 4.34, Corollary 4.35] to show that Generalized Dedekind completeness holds.

Theorem 6.23 Axiom 2.32 holds in $\mathcal{E}$.

Proof The interpretation $\bar{A}(a)$ of $A(x)$, with $a \in{ }^{*} \mathbb{R}$, is a bounded formula. Hence one can apply the Reduction Algorithm to $\bar{A}(a)$ to obtain an equivalent formula of the form $\forall u \in U \exists v \in V B(u, v, a)$, with $U, V \in Z$ and $B$ internal. Since $\bar{A}(a)$ defines a lower halfline, by [2, Thm 4.33] this formula can be reduced to a formula of the form $\exists y \in Y C(y, a)$ or $\forall y \in Y C(y, a)$, with $Y \in Z$ and $C$ internal. Then the result follows by [2, Theorem 4.34, Corollary 4.35].

We show now that the two axioms on multiplication of magnitudes hold in the model $(\mathcal{E},+, \cdot, \leq)$. We recall that magnitudes are interpreted by convex groups. Next proposition states that the interpretation of an idempotent magnitude larger than 1 is a ring with unity, the interpretation of an ideal in a solid is an ideal in the algebraic sense and that under these interpretations the product of an idempotent magnitude and its maximal ideal is equal to this maximal ideal.

Proposition 6.24 Let $S$ be a complete solid. Let $J \in S$ be an idempotent magnitude such that $1<J$. Let $I$ be an ideal of $J$. In the model $(\mathcal{E},+, \cdot, \leq)$, the interpretation $\bar{J}$ of $J$ is a ring and the interpretation $\bar{I}$ of $I$ is an ideal of the ring $\bar{J}$. Moreover, if $I$ is maximal, then $\bar{I}=\left\{1 / x\left|x \in{ }^{*} \mathbb{R}, J<\right| x \mid\right\} \cup\{0\}$ is maximal and $\bar{I} \bar{J}=\bar{I}$. 
Proof The interpretation $\bar{J}$ of $J$ in $\mathcal{E}$ is an idempotent neutrix, which is clearly a ring. An ideal in the sense of Definition 5.2 is a magnitude, so $\bar{I}$ is a neutrix. Because for all $y<J$ one has $y I \leq I$, by the Minkowski definition of the product $x z \in \bar{I}$ for all $x \in \bar{I}$ and $z \in \bar{J}$. This means that $\bar{I}$ is an ideal of $\bar{J}$ in the sense of rings.

Assume now that $I$ is maximal, then $I=\sup \{1 / \omega \mid \omega$ precise, $J<|\omega|\}$ by Theorem 5.3. Let $K \equiv\left\{1 / x\left|x \in{ }^{*} \mathbb{R}, \bar{J}<\right| x \mid\right\} \cup\{0\}$. We show that $\bar{I}=K$. Suppose that there exists $y \in \bar{I} \backslash K$. Then $|1 / y|<\bar{J}$. Hence there exists $u<I$ such that $1 / u<J$, in contradiction with the definition of $I$. Hence $\bar{I} \subseteq K$. Suppose that there exists $z \in K \backslash \bar{I}$. Then $1 / z<\bar{J}$, ie $1 / z \in \bar{J}$, in contradiction with the definition of $K$. Hence $K \subseteq \bar{I}$ and we conclude that $\bar{I}=K$. Suppose the ring $\bar{J}$ has an ideal $L$ with $\bar{I} \subset L \subset \bar{J}$. Let $x \in L \backslash \bar{I}, x<1$ be positive. Because $\bar{I}=K$ we may find $y \in \bar{J} \backslash L$ such that $1 / x<y$. Then $y^{2} \in \bar{J}$. But $x y^{2} \notin L$, since $y<x y^{2}$. So we have a contradiction. As a consequence $\bar{I}$ is the maximal ideal of the ring $\bar{J}$.

As observed above, $y z \in \bar{I}$ for all $y \in \bar{J}$ and $z \in \bar{I}$. Again by the Minkowski definition of the product, it holds that $\bar{I} \bar{J} \subseteq \bar{I}$. Clearly $\bar{I} \subseteq \bar{I} \cdot 1 \subseteq \bar{I} \bar{J}$. Hence $\bar{I} \bar{J}=\bar{I}$.

Corollary 6.25 The structure $(\mathcal{E},+, \cdot, \leq)$ satisfies Axiom 2.30 .

In the syntactical setting of (bounded) IST Axiom 2.31 is verified using an argument based on the logarithm and the exponential function (Koudjeti and Van den Berg [20, Theorem 7.4.4]). It can be adapted without difficulty to our semantic setting.

Theorem 6.26 The structure $(\mathcal{E},+, \cdot, \leq)$ satisfies Axiom 2.31 .

Finally we prove that the axioms on the existence and behavior of natural numbers hold in $\mathcal{E}$.

Theorem 6.27 Let $N$ be interpreted by ${ }^{*} \mathbb{N}$, the set of non-negative nonstandard integers of $* \mathbb{R}$. Then Axioms 2.33-2.35 hold in $\mathcal{E}$.

Proof We interpret the symbol + by the addition in $\mathcal{E}$, the symbol $\cdot$ by the multiplication in $\mathcal{E}$ and the symbol $\leq$ by the order relation in $\mathcal{E}$. This corresponds with the addition ${ }^{*}+$, the multiplication ${ }^{*}$. and the order relation ${ }^{*} \leq$ in $* \mathbb{N}$. Then Axiom 2.33 holds because ${ }^{*} \mathbb{N}$ does not contain negative numbers, ${ }^{*} 0 \in{ }^{*} \mathbb{N}$ and whenever $n \in{ }^{*} \mathbb{N}$, $n+1 \in{ }^{*} \mathbb{N}$, but $y \notin{ }^{*} \mathbb{N}$ for any $y \in{ }^{*} \mathbb{R}$ with $n<y<n+1$. Axiom 2.34 states that induction is valid for each formula $A$ with the symbols $0,1,+$ and $\cdot$, and precise variables which have the property $N$. Then its interpretation ${ }^{*} A$ is a formula with the symbols ${ }^{*} 0,{ }^{*} 1,{ }^{*}+$ and ${ }^{*}$, , with parameters interpreted by elements of $* \mathbb{N}$, and 
quantifications ranging over $* \mathbb{N}$. Because $* \mathbb{N}$ is a model of Peano Arithmetic, Axiom 2.34 indeed holds in $\mathcal{E}$. As regards to Axiom 2.35, it follows from Lemma 3.8 that it is enough to show that the axiom holds for precise elements. Let $x, y \in{ }^{*} \mathbb{R}$ be such that $0<x<y<M$. By construction, for all $a \in{ }^{*} \mathbb{R}$ there exists $n \in{ }^{*} \mathbb{N}$ such that $a<n$. In particular there exists $m \in^{*} \mathbb{N}$ such that $y / x<m$. Hence $y<m x$, so Axiom 2.35 holds in $\mathcal{E}$.

Theorem 6.28 The structure $(\mathcal{E},+, \cdot, \leq)$ satisfies Axioms 2.1-2.35.

Proof Directly from Theorem 6.10, Theorem 6.23, Corollary 6.25, Theorem 6.26 and Theorem 6.27.

Corollary 6.29 Axioms $2.1-2.35$ are consistent with $Z F C$.

\section{Complete arithmetical solids}

Definition 7.1 A model $E$ for Axioms 2.1-2.35 will be called a complete arithmetical solid. The set of magnitudes of $E$ will be denoted by $\mathcal{N}_{E}$. The set of precise numbers of $E$ will be denoted by $\mathcal{P}_{E}$. If there is no ambiguity we drop the subscript $E$ and write simply $\mathcal{N}$, respectively $\mathcal{P}$.

In the previous section we showed that the structure $\mathcal{E}$ given by Definition 6.3 is in fact a complete arithmetical solid. This structure was based on the superstructure $Z$ over the set of real numbers $\mathbb{R}$ and on the nonstandard model ${ }^{*} \mathbb{R}$ of an ultralimit ${ }^{*} Z$ of $Z$. Its set of magnitudes was given in Definition 6.1 and its set of precise numbers was ${ }^{*} \mathbb{R}$. Even if a set of magnitudes is specified in the above way it is to be expected that the set of precise numbers is not uniquely determined. Indeed, we would then have a first-order characterization of the (nonstandard) set of real numbers, for the axioms of Section 2 are stated within first-order logic. However, we will show that the set of non-precise numbers is completely determined. This will be a consequence of Theorem 7.8 which states that if a set of magnitudes is specified in a complete arithmetical solid, the set of non-precise numbers is completely determined as sums of nonstandard rationals and a nonzero magnitude.

For the set of precise numbers we obtain lower and upper bounds. Indeed, Theorem 7.17 states that the set of precise numbers is necessarily a nonstandard ordered field situated between the nonstandard rationals and the nonstandard reals; the field is Archimedean for the corresponding set of nonstandard natural numbers. This is to be compared with 
the well-known theorem saying that an Archimedean ordered field lies between the rationals and the reals. The "standard" structure related to this field is also situated between rationals and reals.

It will be shown that the precise elements of a complete arithmetical solid satisfy the axioms of ZFL (Lutz [22]). The theory ZFL ("Zermelo-Fraenkel-Leibniz") is basically a calculatory nonstandard axiomatics in which the Leibniz rules hold. In Callot [6] it is shown that $Z F L$ is sufficient to develop a nonstandard Calculus in terms of $S$-continuity, $S$-differentiability and $S$-integrability. The axiomatics $Z F L$ is weaker than Nelson's arithmetical axiomatics of Radically Elementary Probability Theory [24] due to the lack of the axiom scheme of External Induction. This axiomatics is here called REPT. In $R E P T$ the axiom scheme of External Induction holds for formulas on the language $\{\mathrm{st}, \in\}$. Nelson shows that it is possible to do advanced stochastics in REPT. We show that in a complete arithmetical solid External Induction holds for formulas in the language $\{\mathrm{st},+, \cdot\}$.

In Subsection 7.1 we show that the algebraic axioms alone are not sufficient to characterize the external numbers by exhibiting a proper substructure ${ }^{\rho} \mathcal{E}$ of $\mathcal{E}$ satisfying all the algebraic axioms. This justifies the introduction of the arithmetical axioms.

In Subsection 7.2 we show that every complete arithmetical solid contains a copy of a nonstandard model of Peano arithmetic. As a consequence, in our framework we have a copy of the nonstandard rationals. By analogy to the construction of the reals via Dedekind cuts we show in Subsection 7.3 that the precise numbers of a complete arithmetical solid are situated between the nonstandard rationals and the nonstandard reals. The proof that a complete arithmetical solid has two built-in models of the rational numbers uses a notion of standard part, here called shadow, and is based on the well-known construction of the standard reals as the quotient of the rationals by the infinitesimals. As a consequence, a complete arithmetical solid $E$ can only be constructed in a nonstandard setting. In Subsection 7.4 we compare our axiomatics with the nonstandard axiomatics $Z F L$ and $R E P T$.

The results in this section suggest that our axiomatic approach gives rise to an alternative way to build nonstandard real numbers, sharing the algebraic spirit of Benci and Di Nasso [1].

It is useful to identify magnitudes $f$ of a solid $S$ with the set $P_{f}$ of its precise elements, ie

$$
P_{f} \equiv\{x \in S|e(x)=0 \wedge| x \mid \leq f\} .
$$

With some abuse of language the sets $P_{f}$ will also be called magnitudes. 
Proposition 7.2 Let $S$ be a solid. Let $X$ be the set of all magnitudes in $S$ and $P(S)$ be the set of all subsets of $S$. Let $\phi: X \rightarrow P(S)$ be the map defined by $\phi(f)=P_{f}$, where $P_{f}$ is given by (8). Then $P_{f}$ is a convex subgroup of $S$ for the addition and order relation of $S$. The map $\phi$ is 1-1.

Proof It is clear that $P_{f}$ is a convex subgroup of $S$ for the addition and order relation of $S$. To prove that $\phi$ is $1-1$, assume that $f, g \in X$ with $f<g$. Then there exists a precise element $p$ such that $f<p<g$. Then $p \in P_{g}$ and $p \notin P_{f}$. Hence $P_{f} \subset P_{g}$.

With some abuse of language, we identify $£$ with the set $P_{£}$ and $\oslash$ with the set $P_{\oslash}$. Elements of $£$ are called limited and elements of $\oslash$ are called infinitesimal.

\subsection{The solid ${ }^{\rho} \mathcal{E}$}

We show that the algebraic axioms alone are not sufficient for a characterization of the external numbers. We do this by exhibiting a proper substructure ${ }^{\rho} \mathcal{E}$ of $\mathcal{E}$ that also satisfies all the algebraic axioms. In this way we also obtain that the symbols $\oslash$, respectively $£$ as defined in (2) may have an interpretation different from the infinitesimals, respectively the limited numbers.

Indeed, let $\rho \in{ }^{*} \mathbb{R}, \rho>0$ be infinitely large. We define $G=\bigcup_{n \in \mathbb{N}}\left[-\rho^{n}, \rho^{n}\right]$ and $H=\bigcap_{n \in \mathbb{N}}\left[-(1 / \rho)^{n},(1 / \rho)^{n}\right]$. Clearly $G$ and $H$ are idempotent. The field $G / H \equiv{ }^{\rho} \mathbb{R}$ was studied by Lightstone and Robinson in [21].

Definition 7.3 We define ${ }^{\rho} \mathcal{I}$ as the set of all convex sets $I \subseteq{ }^{*} \mathbb{R}$ of the form $\bigcup_{n \in \mathbb{N}}\left[-p_{n}, p_{n}\right]$ or $\bigcap_{n \in \mathbb{N}}\left[-1 / p_{n}, 1 / p_{n}\right]$, with $p_{n}>0$, and $p_{n+1} / p_{n}$ increasing such that

$p_{0}=1, p_{1} \geq \rho$ and $p_{n+1} / p_{n} \geq p_{n}^{1 / n}$ for all $n \in \mathbb{N}, n>0$. We let ${ }^{\rho} \mathcal{N}$ be the set of all neutrices of the form $q I$ where $q \in{ }^{*} \mathbb{R}$ and $I \in{ }^{\rho} \mathcal{I}$, and ${ }^{\rho} \mathcal{E}$ as the set of elements of $\mathcal{E}$ of the form $r+L$ where $r \in{ }^{*} \mathbb{R}$ and $L \in{ }^{\rho} \mathcal{N} \cup\{0\} \cup\left\{{ }^{*} \mathbb{R}\right\}$.

Proposition 7.4 The set ${ }^{\rho} \mathcal{I}$ consists of idempotent neutrices, with minimal element greater than 1 equal to $G$ and maximal element less than 1 equal to $H$. Moreover, ${ }^{\rho} \mathcal{I}$ is closed under addition and multiplication and satisfies Axiom 2.30.

Proof Let $J$ be of the form $\bigcup_{n \in \mathbb{N}}\left[-p_{n}, p_{n}\right]$ and $I$ of the form $\bigcap_{n \in \mathbb{N}}\left[-1 / p_{n}, 1 / p_{n}\right]$, with $\left(p_{n}\right)_{n \in \mathbb{N}}$ as given by Definition 7.3. Then $p_{n+1} / p_{n} \geq \rho$ for all $n \in \mathbb{N}, n>0$, which 
implies that both $I$ and $J$ are neutrices. Also $p_{n}^{2} \leq p_{2 n}$ for all $n \in \mathbb{N}, n>0$, which implies that both $I$ and $J$ are idempotent. The set ${ }^{\rho} \mathcal{I}$ is closed under addition, because its elements are neutrices. Then ${ }^{\rho} \mathcal{I}$ is closed under multiplication by Theorem 5.9. Because $p_{n} \geq \rho^{n}$ for all $n \in \mathbb{N}$, one has $J \supseteq G$ and $I \subseteq H$. So $G$ is the minimal element of ${ }^{\rho} \mathcal{I}$ greater than 1 , and $H$ is the maximal element of ${ }^{\rho} \mathcal{I}$. less than 1 . Also $I=\left\{1 / x\left|x \in{ }^{*} \mathbb{R}, J<\right| x \mid\right\} \cup\{0\}$. Then Proposition 6.24 implies that $I$ is the maximal ideal of the ring $J$ and that $I J=I$. Hence ${ }^{\rho} \mathcal{I}$ satisfies Axiom 2.30.

Proposition 7.5 The $\operatorname{set}^{\rho} \mathcal{E}$ satisfies Axioms 2.1-2.31.

Proof All universal axioms hold because ${ }^{\rho} \mathcal{E}$ is a substructure of $\mathcal{E}$. Let $\alpha=a+A \in{ }^{\rho} \mathcal{E}$, then $-\alpha=-a+A \in{ }^{\rho} \mathcal{E}$, so Axiom 2.4 holds. If $\alpha$ is zeroless, one has $A / a^{2} \in \mathcal{N}$, so $1 / \alpha=1 / a+A / a^{2} \in{ }^{\rho} \mathcal{E}$. Hence Axiom 2.9 is verified. Let $B \in{ }^{\rho} \mathcal{N}$. Then $\alpha B=a A+A B$. Because ${ }^{\rho} \mathcal{N}$ is closed under multiplication and addition and the product of a hyperreal and a neutrix is a neutrix it follows that Axiom 2.19 is verified. Axioms 2.24, 2.26 and 2.28 are satisfied by construction, with $m=\{0\}$ and $M={ }^{*} \mathbb{R}$. Because $1=1+\{0\}$, Axiom 2.25 is satisfied. Axiom 2.27 is satisfied because $G \in{ }^{\rho} \mathcal{E}$. Let $J, K \in{ }^{\rho} \mathcal{N}$ with $J<K$. Then $J \subset K$. Any positive precise element $p \in K \backslash J$ satisfies $J<p<K$. Hence, Axiom 2.29 is satisfied. Axiom 2.30 holds by Proposition 7.4. Finally, Axiom 2.31 holds by construction.

By Proposition 7.4, the symbol $\oslash$ can be interpreted by $H$, and the symbol $£$ can be interpreted by $G$. Indeed, in ${ }^{\rho} \mathcal{E}$ the set of neutrices less than 1 has a weak supremum, in fact a maximum, in the form of $H$, while $G$ is the weak infimum (minimum) of the set of neutrices larger than 1 .

To show whether the Generalized Dedekind completeness axiom holds in ${ }^{\rho} \mathcal{E}$, one should establish that definable lower halflines in ${ }^{\rho} \mathcal{E}$ have a weak supremum. This would require a deeper study of polynomials of external numbers, which falls outside of the scope of this article; observe that, due to the fact that distributivity does not hold in full generality, the product of polynomials does not need to be a polynomial. However, the introduction of natural numbers via the arithmetical axioms permits to distinguish between $\mathcal{E}$ and ${ }^{\rho} \mathcal{E}$. Indeed, as will be shown below, the set $\mathcal{E}$ contains a copy of ${ }^{*} \mathbb{N}$, and induction holds in ${ }^{*} \mathbb{N} \cap £$, but not in ${ }^{*} \mathbb{N} \cap G$. For example, in ${ }^{*} \mathbb{N} \cap G$ the domain of function $x \mapsto 2^{x}$ is closed under the successor function, but this function is not total.

\subsection{On induction in complete arithmetical solids}

The interpretation of $N$ in a complete arithmetical solid will be denoted by ${ }^{*} \mathcal{K}$. We show that ${ }^{*} \mathcal{K}$ satisfies the axioms of Peano Arithmetic. Let $A$ be a formula of the 
language of Peano Arithmetic which we denote by $\mathbf{L}=\{\mathbf{0}, \mathbf{1},+, \bullet, \mathbf{s}\}$. We may extend the language $L=\{+, \cdot, \leq\}$ to a language $L^{\prime}$ which includes the symbols $m, u, S c$, corresponding respectively to $\mathbf{0}, \mathbf{1}, \mathbf{s}$. Indeed, let $m$ be the neutral element for addition as in Axiom 2.24. It is easy to see that it is unique and therefore definable. The same is true for the neutral element for multiplication $u$ of Axiom 2.25. Putting $S c(x)=x+u$, we obtain a definable successor function (functional relation) of one variable $S c$. In this way, the formula $A$ has a 1-1 correspondence with a formula $B^{\prime}$ in the extended language $L^{\prime}$ which may be seen as an abbreviation of a formula $C^{\prime}$ of the original language $L$. We let $B$ be the relativization of $B^{\prime}$ to $N$, and $C$ be the relativization of $C^{\prime}$ to $N$. Then within a complete arithmetical solid $E$ the interpretations of $A, B$ and $C$ are all the same.

Theorem 7.6 Let $E$ be a complete arithmetical solid. Then ${ }^{*} \mathcal{K}$ satisfies the Peano Axioms as formulated in the language $\mathbf{L}$.

Proof Observe that all elements of ${ }^{*} \mathcal{K}$ are precise because the predicate $N$ only applies to precise variables. With some abuse of language let 0 be the neutral element of $E, 1$ be the unity of $E$, + the addition on $E$ and $\cdot$ the multiplication on $E$. We may interpret $S c$ by the function $\sigma:{ }^{*} \mathcal{K} \rightarrow{ }^{*} \mathcal{K}$ given by $\sigma(k)=k+1$. As observed above 0 is the interpretation of $\mathbf{0}, 1$ is the interpretation of $\mathbf{1},+$ is the interpretation of,$+ \cdot$ is the interpretation of $\bullet$ and $\sigma$ is the interpretation of $\mathbf{s}$. By Axiom 2.33, $0 \in{ }^{*} \mathcal{K}$ and $1 \in{ }^{*} \mathcal{K}$. Also by Axiom 2.33 the function $\sigma$ is well-defined because if $k \in{ }^{*} \mathcal{K}$ then $k+1 \in{ }^{*} \mathcal{K}$, and all elements of ${ }^{*} \mathcal{K}$ are non-negative. Because 1 is precise, from $\sigma(k)=\sigma\left(k^{\prime}\right)$ we derive that $k=k^{\prime}$ for all $k, k^{\prime} \in{ }^{*} \mathcal{K}$, hence $\sigma$ is 1-1. By Axiom 2.24 one has $k+0=k$ for all $k \in{ }^{*} \mathcal{K}$. By associativity it holds that $k+\sigma\left(k^{\prime}\right)=k+\left(k^{\prime}+1\right)=\left(k+k^{\prime}\right)+1=\sigma\left(k+k^{\prime}\right)$ for all $k, k^{\prime} \in{ }^{*} \mathcal{K}$. By Dinis and Van den Berg [10, Proposition 3.5] one has that $k .0=0$ for all $k \in E$. It follows from the fact that distributivity holds for precise elements that $k . \sigma\left(k^{\prime}\right)=k\left(k^{\prime}+1\right)=k k^{\prime}+k$, for all $k, k^{\prime} \in{ }^{*} \mathcal{K}$. Let $A(x)$ be a property of the language of Peano Arithmetic allowing for a free variable $x$ such that $A(0)$ holds and for all $x$ if $A(x)$ then $A(\mathbf{s}(x))$. As argued before $A$ corresponds to a formula $B$ of the language $L$, relativized to $N$, which has the same interpretation $I$ in $E$. Then $I$ is a subset of ${ }^{*} \mathcal{K}$ such that $0 \in I$ and whenever $k \in I$ one has $k+1 \in I$. Now $\forall x(N(x) \rightarrow B(x))$ by Axiom 2.34. Because the interpretation of $N$ is ${ }^{*} \mathcal{K}$ it follows that $I={ }^{*} \mathcal{K}$. Hence $A(x)$ holds for all $x \in{ }^{*} \mathcal{K}$, ie induction holds for the formula $A$. We conclude that ${ }^{*} \mathcal{K}$ is a model for Peano Arithmetic.

By Theorem 7.6, induction in ${ }^{*} \mathcal{K}$ holds for formulas of $L^{\prime}$ with only precise variables, all relativized to $N$. The set of limited elements of ${ }^{*} \mathcal{K}$ will be denoted by $\mathcal{K}$. We show 
below that $\mathcal{K}$ is also a model of Peano Arithmetic, with induction being valid for all formulas $A(x)$ of $L^{\prime}$ allowing for a free precise variable $x$ and quantifications only over precise variables, possibly with (non precise) parameters. The two types of induction may be compared with Internal Induction and External Induction of IST, the first valid for internal formulas, ie formulas in the language $\{\in\}$, and the second valid also for external formulas, ie formulas in the language $\{\in, \mathrm{st}\}$.

Theorem 7.7 Let $E$ be a complete arithmetical solid. Then $\mathcal{K}$ satisfies the Peano Axioms with induction over formulas $A(x)$ allowing for a free precise variable $x$ and quantifications only over precise variables expressed with the symbols,$+ \cdot$ possibly with (non precise) parameters. In fact ${ }^{*} \mathcal{K}$ is an end extension of $\mathcal{K}$.

Proof By construction $£$ contains 0 and 1 . Because it is an idempotent magnitude it is closed under addition and multiplication. The successor function may be interpreted by the function $s: \mathcal{K} \rightarrow \mathcal{K}$ given by $s(k)=k+1$. Then the Peano Axioms, except induction, are proved along the lines of Theorem 7.6. Let $A(x)$ be a property in the language $L^{\prime}$ allowing for a free precise variable $x$ and quantifications only over precise variables, possibly with (non precise) parameters, such that $A(0)$ holds, and for all $x$, if $A(x)$ then $A(x+1)$. Then $A$ is interpreted by a set $\bar{A}$ with $0 \in \bar{A}$ and whenever $k \in \bar{A}$ one has $k+1 \in \bar{A}$. We prove that $\mathcal{K} \subseteq \bar{A}$, ie induction over $\mathcal{K}$ holds for the formula $A$. Let $B=\{x \mid e(x)=0 \wedge \exists a \in \bar{A}(0 \leq x \leq a)\}$. Let $\gamma=c+C=\operatorname{zup} B$. Then $0 \leq \gamma$ and we may assume that $0 \leq c$. Because $\bar{A}$ is closed under addition by 1 it is impossible that $C \subseteq \oslash$, hence $£ \subseteq C$. Assume $\gamma$ is a supremum. Then $c+C \subseteq B$. Hence $\mathcal{K}={ }^{*} \mathcal{K} \cap £ \subseteq{ }^{*} \mathcal{K} \cap[0, c+£) \subseteq{ }^{*} \mathcal{K} \cap[0, c+C)=\bar{A}$. Assume $\gamma$ is not a supremum. Then $B=[0, c+C[[$ with $0<c+C$. We have $c / 2<c+C$ by Lemma 3.6. Suppose that $c / 2 \in £$. Then $c \in £$. Hence $B \subset £$. We conclude that $C \subseteq \oslash$, a contradiction. Hence $\mathcal{K}={ }^{*} \mathcal{K} \cap £ \subseteq{ }^{*} \mathcal{K} \cap[0, c / 2] \subseteq{ }^{*} \mathcal{K} \cap B=\bar{A}$. It follows that induction over $\mathcal{K}$ holds for the formula $A$. Hence $\mathcal{K}$ is a model for Peano Arithmetic. By construction ${ }^{*} \mathcal{K}$ is an end extension of $\mathcal{K}$.

Given a complete arithmetical solid $E$, we denote by ${ }^{*} \mathcal{Q}$ the set of rational numbers constructed in the usual way from ${ }^{*} \mathcal{K}$ and by $\mathcal{Q} \subset{ }^{*} \mathcal{Q}$ the set of rational numbers constructed from $\mathcal{K}$.

\subsection{Precise and non-precise elements of a complete arithmetical solid}

We start by showing that the non-precise elements of a complete arithmetical solid are characterized by sums of rationals and magnitudes. 
Theorem 7.8 Let $E$ be a complete arithmetical solid and $\tilde{E}$ be the set of non-precise elements of $E$.

(1) For all $p \in \mathcal{P}$ and $N \in \mathcal{N}, N \neq 0$ there exists $q \in{ }^{*} \mathcal{Q}$ such that $p+N=q+N$.

(2) $\tilde{E}=\left\{q+N \mid q \in{ }^{*} \mathcal{Q} \wedge N \in \mathcal{N} \backslash\{0\}\right\}$.

Proof 1. Let $\xi \in \tilde{E}$. Then there exists $p \in \mathcal{P}$ and $N \in \mathcal{N}$ such that $\xi=p+N$. Let $0<b<N$. Then ${ }^{*} \mathcal{Q} \cap £ \cap[p, p+b] \neq \emptyset$. Let $q \in{ }^{*} \mathcal{Q} \cap £ \cap[p, p+b]$. Then $q+N=p+N$.

2. This is a consequence of Theorem 7.8.1.

By the previous theorem, the set of magnitudes of a complete arithmetical solid $E$ determines the set of non-precise elements. However, the nonstandard rationals ${ }^{*} \mathcal{Q}$ give only a lower bound for the precise elements. We will show below that an upper bound is given by nonstandard reals. Also, Theorem 7.8 allows to define standard precise numbers. To see this we need to introduce some definitions and notation.

Definition 7.9 Let $x \in E$ be precise and limited. Then $x+\oslash$ is called the $\mathcal{K}$-shadow of $x$. The $\mathcal{K}$-shadow of a subset $D$ of precise elements of $E$ is the set of $\mathcal{K}$-shadows of all limited elements of $D$. We denote the $\mathcal{K}$-shadow of $\mathcal{Q}$ by $\mathfrak{Q}$, the $\mathcal{K}$-shadow of ${ }^{*} \mathcal{Q}$ by $\mathfrak{F}$ and the $\mathcal{K}$-shadow of $\mathcal{P}$ by $\mathfrak{P}$.

Definition 7.10 Let $K$ be a model of Peano Arithmetic. An ordered field $F \supseteq K$ is $K$-Archimedean if for all $a, b \in F, a, b>0$ there is $k \in K$ such that $k a>b$. A $K$-real field is an ordered field which is $K$-Archimedean and such that every precise lower halfline has a least upper bound.

If $\mathcal{K}=\mathbb{N}$ or $K=\mathbb{N}$, we may suppress the prefix in the above notions, for they correspond to common notions. Observe that a $\mathbb{N}$-real field is isomorphic to $\mathbb{R}$ and that the $\mathbb{N}$-shadow of a limited real number is in 1-1 correspondence with the usual shadow ${ }^{\circ} x$ of $x$, more commonly called standard part (Diener and Diener [8]).

The proof that $\mathcal{P}$ is contained in a copy of the nonstandard reals uses the construction based on lower halflines given by Theorem 7.12. As regards the $K$-shadow of $\mathcal{P}$, we have $\mathfrak{P}=\mathfrak{F}$ by Theorem 7.8. Also, $\mathfrak{Q} \subseteq \mathfrak{P}$. For $\mathcal{K}=\mathbb{N}$ we have $\mathcal{Q}=\mathbb{Q}$, and $\mathfrak{P}$ itself is a real field. Indeed $\mathfrak{P}$ is isomorphic to the quotient of ${ }^{*} \mathbb{Q}$ by the infinitesimals, which is isomorphic to $\mathbb{R}$. In general, the set $\mathfrak{F}$ turns out to be a $\mathcal{K}$-Archimedean ordered field, see Theorem 7.15 below. It is not necessarily a $\mathcal{K}$-real field, for it may not be Dedekind complete, since in principle the Dedekind property holds only for definable cuts. Then by Theorem 7.12 it can be extended to a $\mathcal{K}$-real field. 
Definition 7.11 Let $K$ be a model of Peano Arithmetic and $F$ be a $K$ Archimedean ordered field. Similarly to Definition 3.1, a lower halfline $A$ is precise if there is no positive $d$ such that $a+d \in A$ for all $a \in A$. We define $R_{F}$ as the set of precise lower halflines of elements of $F$ without maximal element. For $f \in F$ we define $H(f)=\{x \in F \mid x<f\}$. We put ${ }^{*} \mathcal{R}=R * \mathcal{Q}$ and $\mathfrak{R}=R_{\mathfrak{F}}$.

Clearly a precise lower halfline $A$ is strictly contained in $F$ and for all $k \in K$ there exist $a \in A$ and $c \in F \backslash A$ such that $c-a<1 / k$.

The proof of the construction of a $K$-real field from a $K$-Archimedean ordered field follows roughly the lines of the usual construction of the reals as Dedekind cuts of the rationals.

Theorem 7.12 Let $K$ be a model of Peano Arithmetic and $F$ be a $K$ Archimedean ordered field. Then $R_{F}$ is a $K$-real field. The mapping $H$ is an isomorphism of the field $F$ onto a subfield $H(F) \subseteq R_{F}$.

Proof As for the definition of addition, multiplication and order, the verification of their first-order properties and the fact that $R_{F}$ contains a copy of $F$ via the mapping $H$, one may follow the lines of a textbook proof of the construction of the real numbers from the rationals using cuts, see for instance Rudin [27]. By construction $R_{F}$ is Archimedean for $K$. We show that the least upper bound property is satisfied for precise halflines. Let $A \subset R_{F}$ be a precise lower halfline. Let

$$
b=\bigcup_{a \in A} a .
$$

Let $x \in b$ and $y \in F$ such that $y<x$. Then there exists $a \in A$ such that $x \in a$. Because $a$ is a lower halfline of $F$ one has $y \in a$. Hence $y \in b$. We conclude that $b$ is a lower halfline of $F$. Also, since $a$ does not have a maximal element, there exists $z \in a$ such that $x<z$. Then $z \in b$ with $x<z$, and we see that $b$ does not have a maximal element. We show that $b$ is precise. If not, there exists a positive $d \in F$ such that $z+d \in b$ whenever $z \in b$. Because $A$ is precise there exist $a \in A$ and $c \notin A$ such that $c-a=d$. There exist elements $x, x^{\prime} \in F$ such that $x^{\prime}-x<d$ with $x \in a$ and $x^{\prime} \notin a$. Also there exist elements $y, y^{\prime} \in F$ such that $y^{\prime}-y<d$ with $y \in c$ and $y^{\prime} \notin c$. Note that $y^{\prime} \notin b$ because $y^{\prime} \notin c$ and $c \notin A$. On the other hand $y-x^{\prime}<d=c-a$ and

$$
y^{\prime}-x=\left(y^{\prime}-y\right)+\left(y-x^{\prime}\right)+\left(x^{\prime}-x\right)<3 d .
$$

Now $x \in b$, so $x+3 d \in b$. Then $y^{\prime} \in b$ because $b$ is a lower halfline, a contradiction. Hence $b$ is precise and we conclude that $b \in F$. 
Finally we show that $b$ is the least upper bound of $A$. Let $a \in A$. Then $a \leq b$ because $x \in b$ whenever $x \in F$ and $x \in a$. Suppose that $b^{\prime}<b$. Then there exists $x \in b$ such that $x \notin b^{\prime}$. Then $x \in a$ for some $a \in A$. Then $b^{\prime}<a$, hence $b^{\prime}$ is not an upper bound of $A$.

Theorem 7.13 Let $K$ be a model of Peano Arithmetic and $F$ be a $K$ Archimedean ordered field. Let $Q$ be the set of rational numbers corresponding to $K$. Then $Q$ may be embedded in $F$ and there is a isomorphism between $R_{Q}$ and $R_{F}$.

Proof It is obvious that $Q$ may be embedded in $F$. To see that $R_{Q}$ and $R_{F}$ are isomorphic, define $\phi: R_{F} \rightarrow R_{Q}$ by $\phi(A)=A \cap Q$. To see that $\phi$ is $1-1$, let $A, B \in R_{F}$ with $A \neq B$; we may suppose that $A \subset B$. Then there exists $y \in B$ such that $x<y$ for all $x \in A$. Because $B$ does not have a maximal element there exists $z \in B$ with $y<z$, and because $F$ is $K$-Archimedean there exists $q \in Q$ such that $y<q<z$. Then $q \in \phi(B) \backslash \phi(A)$. Hence $\phi(A) \neq \phi(B)$. To see that $\phi$ is onto, let $B \in R_{Q}$. Put $A=\{x \in F \mid \exists q \in B, x<q\}$. Then $A$ is a lower halfline of $F$ without a maximal element, otherwise there would exist $q \in B$ with $x<q$; then $q \in F$, because $B$ does not have a maximal element. Clearly $A \cap Q=B$. Hence $\phi(A)=B$. It is also obvious that $\phi$ is respects the algebraic operations and the order. We conclude that there is a isomorphism between $R_{Q}$ and $R_{F}$.

As a consequence we obtain that $\mathcal{P}$ is contained in the ${ }^{*} \mathcal{K}$-real field $R_{\mathcal{P}}$, and can be embedded in $R * Q$.

Corollary 7.14 Let $E$ be a complete arithmetical solid. Then $R_{\mathcal{P}}$ is a ${ }^{*} \mathcal{K}$-real field and the mapping $H$ is an isomorphism of the field $\mathcal{P}$ onto a subfield $H(\mathcal{P}) \subseteq{ }^{*} \mathcal{R}$.

We will now prove that $\mathfrak{P}$ is a $\mathcal{K}$-Archimedean ordered field situated between $\mathfrak{Q}$ and a $\mathcal{K}$-real field $\mathfrak{R}$. This will be a consequence of next theorem which states that $\mathfrak{F}$ is a $\mathcal{K}$-Archimedean ordered field.

Theorem 7.15 Let $E$ be a complete arithmetical solid. The set $\mathfrak{F}$ is a $\mathcal{K}$-Archimedean ordered field.

Proof Let $x=p+\oslash, y=q+\oslash \in \mathfrak{F}$. Then $|p|,|q| \in{ }^{*} \mathcal{Q} \cap £$. Hence $x+y=$ $p+q+\oslash \in \mathfrak{F}$ and $x y=(p+\oslash)(q+\oslash)=p q+p \oslash+q \oslash+\oslash \oslash=p q+\oslash \in \mathfrak{F}$, by Lemma 5.13.4 and Theorem 4.4.1. Because $\mathfrak{F}$ is a substructure of $E$ both addition and multiplication are commutative and associative in $\mathfrak{F}$. Then $\mathfrak{F}$ is an abelian group for addition with neutral element $\oslash$ and inverse $-p+\oslash$ because $p+\oslash+(-p+\oslash)=\oslash$. 
By Lemma 5.13.4 one has $p \oslash \leq \oslash$, so $(1 / p) \oslash \leq \oslash$ by Lemma 4.3.2. Then $(1+\oslash)(p+\oslash)=p+\oslash+p \oslash=p+\oslash$ and, using Theorem 4.4.1

$$
(p+\oslash)\left(\frac{1}{p}+\oslash\right)=1+p \oslash+\frac{1}{p} \oslash+\oslash \oslash=1+\oslash .
$$

Then $\mathfrak{F} \backslash\{\oslash\}$ is also an abelian group for multiplication with neutral element $1+\oslash$ and inverse $1 / p+\oslash$ for the element $p+\oslash$. To prove distributivity, let $x=p+\oslash, y=$ $q+\oslash, z=r+\oslash \in \mathfrak{F}$. By Axiom 2.22 we have $x y+x z=x(y+z)+e(x) y+e(x) z$. Now $e(x) y+e(x) z=\oslash(q+\oslash)+\oslash(r+\oslash)=\oslash$. Also

$$
\begin{aligned}
x(y+z) & =(p+\oslash)(q+\oslash+r+\oslash)=(p+\oslash)(q+r+\oslash) \\
& =p(q+r)+p \oslash+(q+r) \oslash+\oslash \oslash \\
& =p(q+r)+\oslash .
\end{aligned}
$$

Then $x y+x z=x(y+z)+\oslash=x(y+z)$.

Hence distributivity holds, so $\mathfrak{F}$ is a field. Because $\mathfrak{F}$ is a substructure of $E$ the order axioms are valid and we conclude that $\mathfrak{F}$ is indeed an ordered field. By construction $\mathfrak{F}$ is Archimedean for $\mathcal{K}$.

Knowing that $\mathfrak{P}=\mathfrak{F}$ is $\mathcal{K}$-Archimedean ordered field, we obtain a corollary to Theorem 7.12 .

Corollary 7.16 Let $E$ be a complete arithmetical solid. Then $\mathfrak{R}$ is a $\mathcal{K}$-real field and the mapping $H$ is an isomorphism of the field $\mathfrak{P}$ onto a subfield $H(\mathfrak{P}) \subseteq \mathfrak{R}$.

Within a complete arithmetical solid we may now characterize the set of precise numbers and its shadow by lower and upper bounds as follows.

Theorem 7.17 Let $E$ be a complete arithmetical solid. Then up to identifications, ${ }^{*} \mathcal{Q} \subset \mathcal{P} \subseteq{ }^{*} \mathcal{R}$ and $\mathfrak{Q} \subset \mathfrak{P}=\mathfrak{F} \subseteq \mathfrak{R}$.

In the natural case where $\mathcal{K}=\mathbb{N}$ we have some simplifications. The set ${ }^{*} \mathcal{R}$ becomes a nonstandard model ${ }^{*} \mathbb{R}$ of the reals and the set ${ }^{*} \mathcal{Q}$ a nonstandard model ${ }^{*} \mathbb{Q}$ of the rationals. The field $\mathfrak{F}$ can be identified with the quotient of the external set of limited rationals ${ }^{*} \mathbb{Q}$ of a nonstandard model ${ }^{*} \mathbb{R}$ of the reals by the infinitesimal rationals. So $\mathfrak{F}$ itself is already Dedekind complete and is isomorphic to $\mathbb{R}$. With these identifications the set of precise elements is a proper extension of $* \mathbb{Q}$ and a cofinal subfield of $* \mathbb{R}$. As a consequence, up to identifications the lower and upper bounds of Theorem 7.17 take the form ${ }^{*} \mathbb{Q} \subset \mathcal{P} \subseteq{ }^{*} \mathbb{R}$ and $\mathfrak{Q} \subset \mathfrak{P}=\mathbb{R}$. 


\subsection{Complete arithmetical solids and nonstandard analysis}

In this final subsection we investigate the relation between the standard structure and the nonstandard structure of a complete arithmetical solid. We show that the precise numbers satisfy the axiomatics $Z F L$ and that the set of nonstandard natural numbers ${ }^{*} \mathcal{K}$ satisfy the axiomatics $R E P T$, with external induction restricted to the language $\{+, \cdot\}$. The language of $Z F L$ is $\{\in, \lim \}$, where lim stands for "limited" and the axioms are all the axioms of $Z F$ and

(1) $\forall x(\lim (x) \Rightarrow x \in \mathbb{R})$

(2) $\lim (1)$

(3) $\forall x \forall y((\lim (x) \wedge y \in \mathbb{R} \wedge|y| \leq x) \Rightarrow \lim (y))$

(4) $\forall x \forall y((\lim (x) \wedge \lim (y)) \Rightarrow(\lim (x+y) \wedge \lim (x y)))$

(5) $\exists x(x \in \mathbb{R} \wedge \neg \lim (x))$

The rules of calculation of Axiom 4 are called the Leibniz rules. For a presentation of the theory $Z F L$ we refer to Callot [6, page 416].

We recall that the language of $R E P T$ is $\{\mathrm{st}, \in\}$ and its axioms are the axioms of $Z F C$ and

(1) $\operatorname{st}(0)$

(2) $\forall n \in \mathbb{N}(\operatorname{st}(n) \rightarrow \operatorname{st}(n+1))$

(3) $\exists \omega \in \mathbb{N}(\neg \mathrm{st}(\omega))$

(4) $\left(\Phi(0) \wedge \forall^{\mathrm{st}} n \in \mathbb{N}(\Phi(n) \rightarrow \Phi(n+1))\right) \rightarrow \forall^{\mathrm{st}} n \Phi(n)$

In the last axiom $\Phi$ is an arbitrary formula, internal or external, and $\forall^{\text {st }} n \in \mathbb{N} \Phi(n)$ is an abbreviation of $\forall n \in \mathbb{N}(\operatorname{st}(n) \rightarrow \Phi(n))$.

Theorem 7.18 Let $E$ be a complete arithmetical solid. Then $\mathcal{P} \cap £$ satisfies the Leibniz rules. Moreover, if we interpret $\mathbb{R}$ by ${ }^{*} \mathbb{R}$ and the limited elements as elements of $\mathcal{P} \cap £$, the set $\mathcal{P}$ is a model of $Z F L$.

Proof Axiom 1 follows because all elements of $\mathcal{P} \cap £$ are elements of $* \mathbb{R}$. Axioms 2,3 and 4 follow from the fact that $1 \in £$ and $£$ is an idempotent neutrix. Axiom 5 follows from Theorem 7.7.

Theorem 7.19 Let $E$ be a complete arithmetical solid. If we interpret the standard numbers by elements of $\mathcal{K}$, the set ${ }^{*} \mathcal{K}$ is a model of REPT with external induction restricted to the language $\{+, \cdot\}$. 
Proof We interpret $\operatorname{st}(n)$ by $n \in \mathcal{K}$. Then the result is a consequence of Theorem 7.7 .

Observe that in the special case where $\mathcal{K}=\mathbb{N}$ we even have external induction in the language $\{\mathrm{st}, \in\}$ and then ${ }^{*} \mathcal{K}$ is a model of $R E P T$.

\section{Acknowledgements}

The first author acknowledges the support of the Fundação para a Ciência e a Tecnologia, Portugal via the grant [SFRH/BPD/97436/2013], Centro de Matemática, Aplicações Fundamentais e Investigação Operacional (Universidade de Lisboa) via the grant FCT [UID/MAT/04561/2013] and EMMAE14 cohort 3 via the grant [2523].

The authors would like to thank Fernando Ferreira (University of Lisbon) for the suggestion to formulate the Axiom of Generalized Dedekind completeness in the form of a scheme, and an anonymous referee for many corrections and suggestions.

\section{References}

[1] V Benci, M Di Nasso, Alpha-theory: An elementary axiomatics for nonstandard analysis, Expositiones Mathematicae 21:4 (2003) 355 - 386; doi: 10.1016/s07230869(03)80038-5

[2] I van den Berg, Nonstandard asymptotic analysis, Lecture notes in mathematics 1249, Springer-Verlag (1987); doi: 10.1007/bfb0077577

[3] I van den Berg, A decomposition theorem for neutrices, Annals of Pure and Applied Logic 161:7 (2010) 851 - 865; doi: 10.1016/j.apal.2009.10.003

[4] I van den Berg, External borders and strongly open sets, from: "Des Nombres et des Mondes", (E Benoit, J-P Furter, editors), Hermann, Paris (2012) 69-86

[5] N de Bruijn, Asymptotic Analysis, North Holland (1961)

[6] J L Callot, Trois leçons d'Analyse Infinitésimale, from: "Le labyrinthe du continu", (J Salanskis, H Sinaceur, editors), Springer-Verlag, Paris (1992) 369-381

[7] J G van der Corput, Introduction to the neutrix calculus, J. Analyse Math. 7 (1959/1960) 281-399; doi: 10.1007/bf02787689

[8] F Diener, M Diener (editors), Nonstandard analysis in practice, Universitext, SpringerVerlag, Berlin (1995); doi: 10.1007/978-3-642-57758-1 
[9] B Dinis, Old and new approaches to the Sorites paradox, to appear in the proceedings of the Annual Scientific Meeting of Thang Long University; arXiv: 1704.00450

[10] B Dinis, I van den Berg, Characterization of distributivity in a solid, Indagationes Mathematicae (2017); doi: 10.1016/j.indag.2017.10.008

[11] B Dinis, I van den Berg, Algebraic properties of external numbers, J. Log. Anal. 3:9 (2011) 1-30; doi: 10.4115/jla.2011.3.9

[12] B Dinis, I van den Berg, On the quotient class of non-archimedean fields, Indagationes Mathematicae 28 (2017) 784 - 795; doi: 10.1016/j.indag.2017.05.001

[13] R Goldblatt, Lectures on the Hyperreals: An Introduction to Nonstandard Analysis, Graduate Texts in Mathematics, 188, Springer, New York (1998); doi: 10.1007/978-14612-0615-6_20

[14] H Gonshor, Remarks on the Dedekind completion of a nonstandard model of the reals, Pacific Journal of Mathematics 118:1 (1985) 117-132; doi: 10.2140/pjm.1985.118.117

[15] J Justino, I van den Berg, Cramer's rule applied to flexible systems of linear equations, Electron. J. Linear Algebra 24 (2012/13) 126-152; doi: 10.13001/1081-3810.1584

[16] V Kanovei, M Reeken, Mathematics in a nonstandard world I, Mathematica Japonica 45:2 (1997) 369-408

[17] V Kanovei, M Reeken, Nonstandard Analysis, Axiomatically, Springer Monographs in Mathematics (2014); doi: 10.1007/978-3-662-08998-9

[18] H J Keisler, J H Schmerl, Making the Hyperreal Line Both Saturated and Complete, Journal of Symbolic Logic 56 (1991) 1016-1025; doi: 10.2178/jsl/1183743748

[19] F Koudjeti, Elements of External Calculus with an application to Mathematical Finance, Ph.D. thesis, Labyrinth publications, Capelle a/d IJssel, The Netherlands (1995)

[20] F Koudjeti, I van den Berg, Neutrices, external numbers, and external calculus, from: "Nonstandard analysis in practice", (F, M Diener, editors), Universitext, Springer, Berlin (1995) 145-170; doi: 10.1007/978-3-642-57758-1

[21] A H Lightstone, A Robinson, Nonarchimedean Fields and Asymptotic Expansions, North-Holland Mathematical Library Vol. 13, Elsevier (1975); doi: 10.1016/s09246509(08)x7021-7

[22] R Lutz, Rêveries infinitésimales, Gazette des mathématiciens 34 (1987) 79-87

[23] E Nelson, Internal set theory: a new approach to nonstandard analysis, Bull. Amer. Math. Soc. 83:6 (1977) 1165-1198; doi: 10.1090/s0002-9904-1977-14398-x

[24] E Nelson, Radically Elementary Probability Theory, Annals of Mathematical Studies, vol. 117, Princeton University Press, Princeton, N. J. (1987)

[25] E Nelson, The syntax of nonstandard analysis, Ann. Pure Appl. Logic 38 (1988) 123-134; doi: 10.1016/0168-0072(88)90050-4 
[26] A Robinson, E Zakon, A Set-Theoretical Characterization of Enlargements, from: "Applications of Model Theory to Algebra, Analysis and Probability", (W Luxemburg, editor), Holt, Rinehart and Winston, New York (1969) 109-122

[27] W Rudin, Principles of Mathematical Analysis, International series in pure and applied mathematics (3rd ed), McGraw-Hill (1976)

[28] K Stroyan, W Luxemburg, Introduction to the Theory of Infinitesimals (1976) Pure and Applied Mathematics, No. 72. Academic Press New York-London; doi: 10.1016/s00798169(08)x6039-8

[29] F Wattenberg, [0, $\infty$ ]-valued, translation invariant measures on $N$ and the Dedekind completion of * R., Pacific J. Math. 90 (1980) 223-247; doi: 10.2140/pjm.1980.90.223

Departamento de Matemática, Faculdade de Ciências da Universidade de Lisboa, Campo Grande, Ed. C6, 1749-016, Lisboa, Portugal.

Departamento de Matemática, Universidade de Évora, Portugal.

bmdinis@fc.ul.pt, ivdb@uevora.pt

Received: 4 May 2017 Revised: 24 July 2017 